\title{
Estimation of heterogeneous aquifer parameters using centralized and decentralized fusion of hydraulic tomography data
}

\author{
A. H. Alzraiee ${ }^{1}$, D. Baú ${ }^{2}$, and A. Elhaddad ${ }^{1}$ \\ ${ }^{1}$ Department of Civil and Environmental Engineering (1372), Colorado State University, Fort Collins, CO 80523, USA \\ ${ }^{2}$ Department of Civil and Structural Engineering, University of Sheffield, Sheffield, S1 3JD, UK
}

Correspondence to: A. H. Alzraiee (ayman.alzraiee@gmail.com)

Received: 10 March 2014 - Published in Hydrol. Earth Syst. Sci. Discuss.: 11 April 2014

Revised: - - Accepted: 14 July 2014 - Published: 27 August 2014

\begin{abstract}
Characterization of spatial variability of hydraulic properties of groundwater systems at high resolution is essential to simulate flow and transport phenomena. This paper investigates two schemes to invert transient hydraulic head data resulting from multiple pumping tests for the purpose of estimating the spatial distributions of the hydraulic conductivity, $K$, and the specific storage, $S_{\mathrm{s}}$, of an aquifer. The two methods are centralized fusion and decentralized fusion. The centralized fusion of transient data is achieved when data from all pumping tests are processed concurrently using a central inversion processor, whereas the decentralized fusion inverts data from each pumping test separately to obtain optimal local estimates of hydraulic parameters, which are consequently fused using the generalized Millman formula, an algorithm for merging multiple correlated or uncorrelated local estimates. For both data fusion schemes, the basic inversion processor employed is the ensemble Kalman filter, which is employed to assimilate the temporal moments of impulse response functions obtained from the transient hydraulic head measurements resulting from multiple pumping tests. Assimilating the temporal moments instead of the hydraulic head transient data themselves is shown to provide a significant improvement in computational efficiency. Additionally, different assimilation strategies to improve the estimation of $S_{\mathrm{s}}$ are investigated. Results show that estimation of the $K$ and $S_{\mathrm{s}}$ distributions using temporal moment analysis is fairly good, and the centralized inversion scheme consistently outperforms the decentralized inversion scheme.
\end{abstract}

\section{Introduction}

A detailed description of hydraulic properties, such as hydraulic conductivity, $K$, and specific elastic storage, $S_{\mathrm{s}}$, of groundwater systems is essential to predict flow and solute transport in porous media. Typically, these properties are inherently heterogeneous, and cannot be determined uniquely using a finite set of sparse measurements. A direct method to map the spatial variability of these properties is based on the collection of a large number of core samples, which are then analyzed in the laboratory to obtain conductivity and storage properties. These methods, however, are laborious, expensive, and time consuming (Butler Jr. et al., 1999). In general, sampling of groundwater system states, such as hydraulic head or solute concentrations, is relatively easier and more cost-effective. Therefore, characterization of the aquifer parameters using system states can be achieved by solving an inverse problem (Sun, 1994; Tarantola, 2004).

Analyses of hydraulic head data resulting from pumping tests (Theis, 1935; Cooper and Jacob, 1946) and slug tests (Butler Jr., 1998) using type-curve techniques are classic examples of inverse methods used to infer hydraulic properties of porous media. In a pumping test, an aquifer is stressed at a well and the response of the hydraulic head field is monitored at a number of observation wells. The resulting data are processed using an analytical solution to obtain a lumped estimate of the transmissivity and the storativity of the aquifer at a scale equal to the radius of the developed cone of depression. While these estimates are useful to guide future groundwater development of an aquifer at a regional scale, they provide little or no information about the local spatial variability of parameters, which is essential, for example, to 
model solute transport processes. In addition, the estimates obtained by pumping tests are shown to be affected by the location of the pumping well and the degree of heterogeneity within the cone of depression (Wu et al., 2005).

A relatively recent alternative method for estimating the spatial distribution of aquifer parameters at a high resolution is hydraulic tomography (HT) (Gottlieb and Dietrich, 1995; Butler Jr. et al., 1999; Yeh and Liu, 2000; Berg and Illman, 2011). In HT, an aquifer is stressed at different locations and the responses to these stresses at a network of observation wells are inverted to map the parameters spatially.

During the last decade, HT has been intensively studied both numerically and experimentally to assess its performance with a few field applications (Straface et al., 2007; Bohling et al., 2007). HT studies have covered several flow conditions, ranging from steady-state flow (Yeh and Liu, 2000) to transient flow (Zhu and Yeh, 2005) in both confined and unconfined aquifers (Cardiff and Barrash, 2011). HT has been applied to joint unconfined and vadose zone flow problems (Mao et al., 2013) and for both 2-D (two-dimensional) (Yeh and Zhu, 2007) and 3-D settings (Cardiff et al., 2012; Illman et al., 2009; Berg and Illman, 2013). A number of sandbox laboratory experiments have been performed to validate HT methods off-site (Liu, 2002; Liu et al., 2007; Illman et al., 2010), which have deemed HT a promising technique for characterizing aquifer properties at high resolution. For instance, Illman et al. (2010) compared various approaches to characterize the $K$ field using a sandbox and found that HT consistently outperformed kriging interpolation of smallscale $K$ measurements. Similar results reported by Illman et al. (2012) revealed that predictions of solute transport are better characterized with estimates from HT surveys in comparison to traditional geostatistical analyses and effective parameters. A comprehensive list of previous HT studies is provided by Cardiff and Barrash (2011).

In HT studies, hydraulic head transient data have been inverted using different algorithms, such as the sequential successive linear estimator (SSLE) (Yeh and Liu, 2000), the quasi-linear approach (Kitanidis, 1995; Liu and Kitanidis, 2011), the Bayesian maximum a posteriori (MAP) approach (Castagna and Bellin, 2009), and the ensemble Kalman filter (EnKF) (Schöniger et al., 2012).

Despite the success in verifying its estimates numerically and experimentally, HT faces two major challenges related to the heavy computational burden associated with the inversion process (Zhu and Yeh, 2005) and the nonuniqueness of the solution of the inverse problem, a situation where infinite possible combinations of input parameters and model structures produce the same model output (Moore and Doherty, 2006). With respect to the latter, Bohling and Butler (2010) caution practicing hydrologists against "overselling" the reliability of HT estimates based on their pilot point inverse method, and argue that some form of regularization is typically necessary to reduce uncertainties associated with the nonuniqueness effect. In this work, HT data are inverted using the EnKF. While not resolving the nonuniqueness issue completely, inversion algorithms based on the EnKF constitute an ideal framework to handle the problem of nonuniqueness resulting from parameter uncertainty only, as opposed to nonuniqueness resulting from uncertainty in conceptual models and process assumptions.

With its roots in Bayesian analysis, the EnKF updates a prior ensemble of possible realizations of system states and parameters based upon collected state measurements, so that the posterior state-parameter ensemble resembles a nonunique set of possible solutions. Therefore, the ensemble mean of the posterior ensemble provides an unbiased estimate of the system parameters. The EnKF offers several other advantages, such as computational efficiency (Franssen and Kinzelbach, 2009), avoiding sensitivity computations, such as those required by the SSLE (Yeh and Liu, 2000), and improved accuracy when using ensemble-based covariance estimations instead of sensitivity-based covariance estimations (Schöniger et al., 2012).

A possible effective approach to improving parameter estimations for ill-posed problems is by integrating data from independent sources, which may be related to different physical processes, such as hydraulic, geophysical, geomechanical, and chemical processes (Bohling and Butler, 2010). In this situation, different physical processes (models) are utilized to relate measured responses to aquifer properties. The inversion of such multi-source data may take two general avenues: centralized fusion (CF) and decentralized fusion (DF). In this work, we investigate and compare the two approaches, one based on $\mathrm{CF}$ and another based on DF, to assimilate transient hydraulic head HT data for the characterization of the $K$ and $S_{\mathrm{s}}$ fields of a confined aquifer. With the $\mathrm{CF}$ method, all data resulting from all experiments are inverted simultaneously using a single "global" EnKF. The DF method, however, assimilates each data set resulting from a single experiment separately using a "local" EnKF to obtain a local estimate of parameters. The multiple local estimates are then "fused" using the generalized Millman formula (GMF) algorithm (Bar-Shalom and Campo, 1986; Shin et al., 2006), which constitutes an unbiased linear estimator of multiple correlated or uncorrelated estimates. The two inversion schemes are implemented to assimilate the responses resulting from five pumping tests. However, the methodology can be generalized to merge multiple parameter estimations resulting from inverting different physical processes.

As mentioned earlier, computational cost constitutes an issue for the application of HT methods for aquifer characterization. Typically, HT-based algorithms require inverting a large amount of transient data resulting from multiple experiments and at multiple observation wells, which produces the so-called "data-overload" problem (Zhu and Yeh, 2005). Assimilation of transient data with the EnKF or the ensemble smoother (ES) (Evensen, 2009) is computationally intensive for two reasons. First, the computation of the forecast ensemble of states and parameters requires simulating transient 
flow for a large number of realizations, which typically involves a considerable computational effort. Second, the resulting spatiotemporal cross-covariance matrix is typically large and difficult to manipulate. In this study, we propose to assimilate temporal moments of the impulse response function of transient drawdown data (Harvey and Gorelick, 1995; Von Asmuth and Maas, 2001; Li et al., 2005; Bakker et al., 2008; Olsthoorn, 2008; Von Asmuth et al., 2008), rather than the hydraulic head data themselves.

In the temporal moment analysis, the original parabolic partial differential equation (PDE) governing groundwater flow is transformed into two simpler and easier to solve Poisson-type PDEs (Zhu and Yeh, 2006; Li et al., 2005). Although it has been shown that inversion of temporal moments provides a drastic reduction in central processing unit (CPU) time and a reliable estimate of the $K$ field, it has also been found to produce an unreliable characterization of the $S_{\mathrm{s}}$ field (Yin and Illman, 2009). In this work, we devise a strategy that can optimize the estimation of the $S_{\mathrm{s}}$ field, while still benefiting from the reduced problem complexity achieved with the temporal moment formulation.

The article is organized as follows. The methodologies of the two inversion schemes are presented in Sect. 2. A description of the numerical experiments used to investigate the inversion approaches is provided in Sect. 3. In Sect. 4, the obtained results are presented and discussed.

\section{Methodology}

In the following, we provide an overall description of the proposed HT approaches, followed by a detailed description of each component of the methodology. For the purpose of estimating the hydraulic parameters $K$ and $S_{\mathrm{s}}$, we assume that a series of separate pumping tests is conducted at $N_{\mathrm{p}}$ wells installed at different locations within a confined aquifer. In each pumping test, the pumping well $i\left(i \in\left\{1,2, \ldots, N_{\mathrm{p}}\right\}\right)$ is operated at the flow rate $Q_{i}$. The resulting transient hydraulic head data, $h_{i j}(t)$, are recorded at number $N_{\mathrm{o}}$ of observation wells $\left(j \in\left\{1,2, \ldots, N_{\mathrm{o}}\right\}\right)$.

The size of such measurement data sets is typically quite large. To reduce the computational requirement associated with the inversion of large amount of temporal data, the hydraulic head hydrographs are used to compute the temporal moments of the impulse response function (IRF) at each observation well $j$, in particular, the zeroth temporal, $m_{0}^{j}$, and the first temporal moments, $m_{1}^{j}$. Procedures followed to calculate the temporal moments of the IRF using the measured hydraulic head are discussed in Sect. 2.1. These temporal moments are treated as observations.

The effect of the spatial variability of the aquifer hydraulic parameters, namely $K$ and $S_{\mathrm{s}}$, on the spatial distribution of the temporal moments of the IRF are achieved by means of moment-generating PDEs, which are discussed in Sect. 2.2. The numerical solution of these equations is also discussed in the same section. Two numerical models - one to predict the zeroth temporal moment $m_{0}$ and another to predict the first temporal moment $m_{1}$ - are employed to simulate an ensemble of randomly generated realizations of the $K$ and $S_{\mathrm{s}}$ fields. At this point, the forecast temporal moments, obtained by solving the moment-generating PDEs numerically, and the observed moments, computed from transient hydraulic head measurements, are available and can be subsequently utilized

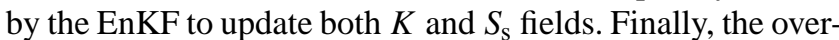
all inversion algorithm is applied either through a CF scheme or a DF scheme, as discussed in Sect. 2.3.

\subsection{Estimation of temporal moments of measured hydraulic head}

In pumping tests, data may be recorded with high temporal frequency or even continuously in time. Assimilating such a large amount of transient data using a Kalman filter (Kalman, 1960) scheme is computationally prohibitive and impractical (Evensen, 2009). Time series analyses allow for shrinking hydrographs of hydraulic head data into low-order temporal moments, which are related to aquifer hydraulic properties through moment-generating partial differential equations. To illustrate, assume that an aquifer system is stressed by a well with a time dependent flow rate $Q(t)$ resulting in transient change in hydraulic head $h(x ; t)$, where the vector $\boldsymbol{x}$ includes the coordinates of the location of an observation well, and $t$ represents time. For linear systems, $h(\boldsymbol{x} ; t)$ can be expressed as a function of $Q(t)$ through a convolution integral (Von Asmuth and Maas, 2001; Li et al., 2005; Bakker et al., 2008; Olsthoorn, 2008; Von Asmuth et al., 2008):

$h(\boldsymbol{x} ; t)=\int_{0}^{t} Q(\tau) \theta(\boldsymbol{x} ; t-\tau) \mathrm{d} \tau$,

where $\theta(\boldsymbol{x} ; t-\tau)$ is the IRF; that is, the response of the aquifer at location $\boldsymbol{x}$ and time $t$ to a unit flow rate impulse at the well at time $\tau$. Accordingly, the objective of time series analysis is to obtain the IRF for every stress source and at each observation well. A possible approach to achieve this is by fitting a parametric function to represent the IRF for each stress source at each observation well (Von-Asmuth et al., 2002; Bakker et al., 2008). Consequently, the obtained IRF function can be used to calculate the $k$ th temporal moment as follows:

$m_{k}(\boldsymbol{x})=\int_{0}^{\infty} t^{k} \theta(\boldsymbol{x} ; t) \mathrm{d} t$.

Alternatively, Li et al. (2005) proposed the following equations for calculating the zeroth moment, $m_{0}$, and the first moment, $m_{1}$, of the IRF using hydraulic head measurements resulting from a constant continuous extraction rate $Q$ : 
$m_{0}(\boldsymbol{x})=\frac{h(\boldsymbol{x} ; 0)-h(\boldsymbol{x} ; \infty)}{Q}$,

$m_{1}(\boldsymbol{x})=\frac{\int_{0}^{\infty}[h(\boldsymbol{x} ; t)-h(\boldsymbol{x} ; \infty)] \mathrm{d} t}{Q}$,

where $h(\boldsymbol{x} ; 0)$ and $h(\boldsymbol{x} ; \infty)$ represent, respectively, the initial and the steady state hydraulic heads at location $\boldsymbol{x}$. Using Eqs. (3) and (4), the observed zeroth temporal moment and the first temporal moment are computed at all observation wells and for each pumping test. In symbolic form, the observed moments from each pumping test can be denoted as $m_{0, i j}$ and $m_{1, i j}\left(i \in\left\{1,2, \ldots, N_{\mathrm{p}}\right\} ; j \in\left\{1,2, \ldots, N_{\mathrm{o}}\right\}\right)$. At this point, the transient-hydraulic head large data set at each observation well is shrunk into the two values $m_{0, i j}$ and $m_{1, i j}$. In the following sections, the numerical simulation of temporal moments is presented.

\subsection{Moment generating equations}

Transient groundwater flow in a saturated heterogeneous porous medium is governed by the PDE:

$\nabla[\boldsymbol{K}(\boldsymbol{x}) \nabla h]+Q(\boldsymbol{x} ; t)=S_{\mathrm{s}}(\boldsymbol{x}) \frac{\partial h}{\partial t}$,

where $\nabla$ is the differential operator, $\boldsymbol{K}$ is the hydraulic conductivity tensor, $S_{\mathrm{s}}$ is the specific elastic storage, and $Q(\boldsymbol{x} ; t)$ represents generic source/sink terms at location $\boldsymbol{x}$ and time $t$. Equation (5) may be solved by imposing Dirichlet boundary conditions $h(\boldsymbol{x} ; t)=h_{\mathrm{D}}(\boldsymbol{x} ; t)$ at a prescribed portion of the domain boundary $\Gamma_{\mathrm{D}}$, Neumann boundary conditions $K(\boldsymbol{x}) \nabla h(\boldsymbol{x} ; t)=q_{\mathrm{N}}(\boldsymbol{x} ; t)$ at another portion of the domain boundary $\Gamma_{\mathrm{N}}$, and initial boundary conditions $h(\boldsymbol{x} ; 0)=h_{0}(\boldsymbol{x})$ throughout the domain.

For a unit impulse extraction $Q(\boldsymbol{x} ; t)=\delta\left(x_{\mathrm{w}}\right)$ at location $x_{\mathrm{W}}$, the $k$ th temporal moment, $m_{k}$, of the IRF of drawdown, $s(\boldsymbol{x} ; t)=h(\boldsymbol{x} ; 0)-h(\boldsymbol{x} ; t)$, might be computed by multiplying Eq. (5) by $t^{k}$ and integrating over the time interval $[0,+\infty)$. The resulting moment-generating equation is $(\mathrm{Li}$ et al., 2005; Yin and Illman, 2009) the following:

$\nabla\left[\boldsymbol{K}(\boldsymbol{x}) \nabla m_{k}\right]+\delta_{k}\left(\boldsymbol{x}_{\mathrm{w}}\right)+k S_{\mathrm{s}}(\boldsymbol{x}) m_{k-1}=0$,

where $\delta_{k}\left(x_{\mathrm{w}}\right)$ is equal to unity if $k=0$ and equal to zero if $k>0$. Similarly, the boundary conditions of the temporalmoment equations are expressed as $m_{k}(\boldsymbol{x})=0$ for the Dirichlet boundary $\Gamma_{\mathrm{D}}$ and $K(\boldsymbol{x}) \nabla m_{k}=0$ for the Neumann boundary $\Gamma_{\mathrm{N}}$.

Because the observations (Sect. 2.1) consist of the zeroth and the first temporal moments, the simulation of only $m_{0}$ and $m_{1}$ is sought. These moments can be obtained by solving numerically the following two PDEs:

$$
\begin{aligned}
& \nabla\left[\boldsymbol{K}(\boldsymbol{x}) \nabla m_{0}\right]+1\left(\boldsymbol{x}_{\mathrm{w}}\right)=0, \\
& \nabla\left[\boldsymbol{K}(\boldsymbol{x}) \nabla m_{1}\right]+S_{\mathrm{s}}(\boldsymbol{x}) m_{0}=0 .
\end{aligned}
$$

Equation (7) is equivalent to a steady-state flow problem characterized by a unit extraction rate, denoted as $1\left(\boldsymbol{x}_{\mathrm{w}}\right)$, at well location $\boldsymbol{x}_{\mathrm{w}}$. Equation (8) is equivalent to a steady-state flow problem with a forcing term constituted by a spatially variable recharge equal to $S_{\mathrm{s}}(\boldsymbol{x}) m_{0}$. Both Eqs. (7) and (8) can be solved using a common groundwater flow simulator, such as the well-known finite-difference model MODFLOW2000 (Harbaugh et al., 2000).

\subsection{Inversion approaches}

This section presents the approaches adopted here to invert the temporal moments in order to characterize the spatial distributions of $K$ and $S_{\mathrm{s}}$. Using a Bayesian framework to pose the inversion problem, the vector of system parameters, $\phi$, can be updated in light of newly collected data $\boldsymbol{m}$ as follows:

$p(\boldsymbol{\phi} \mid \boldsymbol{m}, \boldsymbol{I})=\frac{p(\boldsymbol{m} \mid \boldsymbol{\phi}, \boldsymbol{I}) p(\boldsymbol{\phi}, \boldsymbol{I})}{p(\boldsymbol{m}, \boldsymbol{I})}$,

where $p(\boldsymbol{\phi} \mid \boldsymbol{m}, \boldsymbol{I})$ is the posterior probability distribution function (PDF) of $\boldsymbol{\phi}$ given the measurements $\boldsymbol{m}$ and the generic "prior" information $\boldsymbol{I} ; p(\boldsymbol{m} \mid \boldsymbol{\phi})$ is the likelihood PDF, that is, the probability of the measurements $\boldsymbol{m}$ conditional to the parameters $\boldsymbol{\phi} ; p(\boldsymbol{\phi}, \boldsymbol{I})$ is the prior PDF of $\boldsymbol{\phi}$; and $p(\boldsymbol{m}, \boldsymbol{I})$ is a normalization term. An exact solution to Eq. (9) can be obtained if the measurements $\boldsymbol{m}$ are related to the parameters $\boldsymbol{\phi}$ through a linear relationship, and when all PDFs in Eq. (9) are Gaussian. This solution is widely known as the Kalman filter (KF) (Kalman, 1960).

In the classical implementation of the KF, the data assimilation of state follows a two-stage forecast-update process. In the forecast stage, a forward-in-time prediction of the current state, along with its error covariance is made. The forecast state is then updated as field measurements become available. In this work, the inversion problem is reduced to a timeindependent inversion problem, which means that the forecast stage does not include any forward-in-time prediction. That is to say, the forecast stage is limited to the solution of the equivalent steady-state groundwater problems expressed by Eqs. (7) and (8).

In addition to being limited to Gaussian linear systems, the $\mathrm{KF}$ is computationally expensive when applied to largescale problems. Evensen (1994) expanded the applicability of the KF to nonlinear systems through the EnKF. Within the EnKF, the prior PDFs of the system states are approximated using an ensemble of realizations that characterize the prior uncertainty in the system parameters and states.

\subsubsection{Forecast of parameters and system states}

From the perspective of subsurface flow, the major parameters that typically characterize a groundwater system are the hydraulic conductivity, $K$, and the specific storage, $S_{\mathrm{s}}$. These parameters are inherently heterogeneous and cannot 
be determined uniquely using a finite set of measurements. Therefore, it is convenient to describe these parameters using a geostatistical conceptual model (Matheron, 1962; Isaaks and Srivastava, 1990; Cressie, 1993; Diggle and Ribeiro, 2007), according to which a heterogeneous field is modeled as a spatially distributed random process, characterized by a trend model and a covariance model. In this study, we assume the log-transformed parameters $Y=\ln (K)$ and $Z=\ln \left(S_{\mathrm{s}}\right)$ to fit to two independent isotropic and stationary (with no trend) Gaussian processes (de Marsily, 1986), with prescribed covariance models $C_{Y Y}\left(d ; \sigma_{Y}^{2} ; \lambda_{Y}\right)$ and $C_{Z Z}\left(d ; \sigma_{Z}^{2} ; \lambda_{Z}\right)$, respectively. The scalar $d$ represents the distance between any two points. The parameters $\sigma^{2}$ and $\lambda$ represent variance and the correlation length of each random process. The stationary means of the two fields are denoted as $\mu_{Y}$ and $\mu_{Z}$. A spherical covariance function is assumed for both $C_{Y Y}$ and $C_{Z Z}$ (Deutsch and Journel, 1997). This choice is somewhat arbitrary and other covariance functions might be used to describe the spatial correlation of random fields without altering the general inversion methodology.

Using these geostatistical models, it is possible to generate an ensemble of $N_{\text {ens }}$ equally likely realizations for both $Y$ and $Z$. The ensemble of the natural logarithm of $K$ is obtained as $\mathbf{Y}=\left[\mathbf{Y}_{1}, \ldots, \mathbf{Y}_{N_{\text {ens }}}\right]$, where $\mathbf{Y}_{k} \in R^{n \times 1}$ $\left(k \in\left\{1,2, \ldots, N_{\text {ens }}\right\}\right)$ is a realization of $Y$, and $n$ is the number of cells of the finite-difference grid adopted to discretize the aquifer domain. The ensemble of the natural logarithm of $S_{\mathrm{s}}, \mathbf{Z} \in R^{n \times N_{\text {ens }}}$ is generated in a similar fashion. The resulting ensembles can be seen as discrete approximations of the forecast, or prior, joint PDFs of $Y$ and $Z$.

In the forecast stage, Eqs. (7) and (8) are solved numerically to predict the system states, that is, the temporal moments, in each pumping test. Each realization $\mathbf{Y}_{k}$ in the ensemble $\mathbf{Y}$ is numerically simulated using Eq. (7) to obtain $\boldsymbol{m}_{0, k} \in \mathrm{R}^{n \times 1}$, a vector including the spatial distribution of the zeroth moment at the cells of the finitedifference grid. Next, $\boldsymbol{m}_{0, k}$ and the parameters $\mathbf{Y}_{k}$ and $\mathbf{Z}_{k}$ are used to compute the first-moment vector $\boldsymbol{m}_{1, k} \in R^{n \times 1}$ by solving Eq. (8). Therefore, all realizations of states $\boldsymbol{m}_{0, k}$ and $\boldsymbol{m}_{1, k}\left(k \in\left\{1,2, \ldots, N_{\mathrm{ens}}\right\}\right)$ can be assembled into the $n \times N_{\text {ens }}$ matrices $\mathbf{M}_{0}=\left[\boldsymbol{m}_{0,1}, \ldots, \boldsymbol{m}_{0, N_{\text {ens }}}\right]$ and $\mathbf{M}_{1}=\left[\boldsymbol{m}_{1,1}, \ldots, \boldsymbol{m}_{1, N_{\mathrm{ens}}}\right]$, respectively. To proceed to the update stage, we propose two alternatives: $\mathrm{CF}$ and DF. Schematic diagrams of the two methods are provided in Fig. 1.

\subsubsection{Parameter estimation by centralized fusion}

In the $\mathrm{CF}$ scheme (Fig. 1b), forecast ensembles obtained from simulating independent pumping tests are augmented into a single global forecast matrix $\mathbf{X}_{\mathrm{f}}^{Y}=\left[\begin{array}{llll}\mathbf{Y}, & \mathbf{M}_{0}^{1}, & \ldots, & \mathbf{M}_{0}^{N_{\mathrm{p}}}\end{array}\right]^{T}$, where $\mathbf{M}_{0}^{i}$ represents the zeroth-moment ensemble for the $i$ th pumping test $\left(i \in\left\{1,2, \ldots, N_{\mathrm{p}}\right\}\right)$. Note that the matrix $\mathbf{X}_{\mathrm{f}}^{Y}$ has size $\left(N_{\mathrm{p}}+1\right) n \times N_{\text {ens. }}$. As a matter of fact, there are several (a) Centralized Fusion

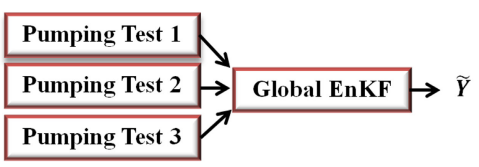

(b) Decentralized Fusion

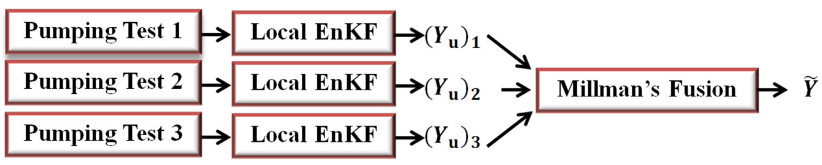

Figure 1. Flowcharts illustrating the structure of (a) the CF approach and (b) the DF approach.

possibilities to assemble the forecast matrix, some of which are listed in Table 1. Formulations A, B, and C provide alternatives for forming $\mathbf{X}_{\mathrm{f}}^{Y}$ in order to estimate the $Y$ field, whereas formulations $\mathrm{D}$ and $\mathrm{E}$ address possible alternatives for estimating the $Z$ field. In Sect. 4 we investigate the implications of employing different formulations of the forecast matrix. Here, we focus exclusively on formulation A in Table 1 to illustrate the $\mathrm{CF}$ procedure.

From the augmented state-parameter forecast matrix $\mathbf{X}_{\mathrm{f}}^{Y}$, the global prior covariance matrix $\mathbf{P}_{\mathrm{f}}^{Y} \in R^{\left(N_{\mathrm{p}}+1\right) n \times\left(N_{\mathrm{p}}+1\right) n}$ can be approximated as

$\mathbf{P}_{\mathrm{f}}^{Y}=\frac{\left(\mathbf{X}_{\mathrm{f}}^{Y}-\overline{\mathbf{X}}_{\mathrm{f}}^{Y}\right) \cdot\left(\mathbf{X}_{\mathrm{f}}^{Y}-\overline{\mathbf{X}}_{\mathrm{f}}^{Y}\right)^{T}}{N_{\mathrm{ens}}-1}$,

where $\overline{\mathbf{X}}_{\mathrm{f}}^{Y}$ is the prior ensemble mean matrix, calculated as $\overline{\mathbf{X}}_{\mathrm{f}}^{Y}=\mathbf{X}_{\mathrm{f}}^{Y} \cdot \mathbf{1}_{N_{\text {ens }}}$ and $\mathbf{1}_{N_{\mathrm{ens}}} \in R^{N_{\text {ens }} \times\left(N_{\mathrm{p}}+1\right) n}$ is a matrix with all elements equal to $1 / N_{\text {ens }}$.

To facilitate the assimilation procedure, measurements collected from $N_{0}$ observations wells and $N_{\mathrm{p}}$ pumping tests are vertically concatenated in a single vector. Therefore, the vector of measurements for the zeroth moment can be denoted as $\boldsymbol{d}_{0}=\left[m_{0, i, j}\right] \in R^{N_{\mathrm{p}} N_{0} \times 1}$, where $i$ is the pumping test index, and $j$ is the observation well index.

Following an EnKF-like procedure, the measurements $\boldsymbol{d}_{0}$ are assimilated to update both systems states and parameters. Therefore, the update state-parameter matrix, $\mathbf{X}_{\mathrm{u}}^{Y} \in R^{\left(N_{\mathrm{p}}+1\right) n \times N_{\text {ens }}}$ and the update covariance matrix, $\mathbf{P}_{\mathrm{u}}^{Y} \in R^{\left(N_{\mathrm{p}}+1\right) n \times\left(N_{\mathrm{p}}+1\right) n}$ can be expressed as follows:

$\mathbf{X}_{\mathrm{u}}^{Y}=\mathbf{X}_{\mathrm{f}}^{Y}+\mathbf{K} \cdot\left(\mathbf{D}_{0}-\mathbf{H} \cdot \mathbf{X}_{\mathrm{f}}^{Y}\right)$,

$\mathbf{P}_{\mathrm{u}}^{Y}=(\mathbf{I}-\mathbf{K} \cdot \mathbf{H}) \cdot \mathbf{P}_{\mathrm{f}}^{Y} \cdot(\mathbf{I}-\mathbf{K} \cdot \mathbf{H})^{T}+\mathbf{K} \cdot \mathbf{R} \cdot \mathbf{K}^{T}$,

where $\mathbf{D}_{0} \in R^{N_{\mathrm{p}} N_{0} \times N_{\text {ens }}}$ is a matrix whose columns are obtained by perturbing the measurement vector $\boldsymbol{d}_{0}$ with a Gaussian zero-mean noise, characterized by the error covariance matrix $\mathbf{R} \in R^{N_{\mathrm{p}} N_{0} \times N_{\mathrm{p}} N_{0}} ; \mathbf{H} \in R^{N_{\mathrm{p}} N_{\mathrm{o}} \times\left(N_{\mathrm{p}}+1\right) n}$ is a matrix that maps each measurement to its location in the finitedifference grid and to its corresponding pumping test. The 
Table 1. Alternative formulations of the forecast matrix investigated in the numerical experiments.

\begin{tabular}{|c|c|c|}
\hline Formulation & Description & Forecast matrix \\
\hline A & $\begin{array}{l}\text { Estimate } K \text { field by assimilating } m_{0} \text { measure- } \\
\text { ments only, with PDE (Eq. 7) as forecast model. }\end{array}$ & $\mathbf{X}_{\mathrm{f}}^{Y}=\left[\mathbf{Y} ; \mathbf{M}_{0}^{1} ; \ldots ; \mathbf{M}_{0}^{N_{\mathrm{p}}}\right]$ \\
\hline B & $\begin{array}{l}\text { Estimate } K \text { field by assimilating } m_{1} \text { measure- } \\
\text { ments only, with PDE (Eq. } 8 \text { ) as forecast model, } \\
\text { in which the } Y \text { forecast ensemble and its corre- } \\
\text { sponding } m_{0} \text { forecast ensemble (obtained from } \\
\text { PDE Eq. 7) are used. }\end{array}$ & $\mathbf{X}_{\mathrm{f}}^{Y}=\left[\mathbf{Y} ; \mathbf{M}_{1}^{1} ; \ldots ; \mathbf{M}_{1}^{N_{\mathrm{p}}}\right]$ \\
\hline $\mathrm{C}$ & $\begin{array}{l}\text { Estimate } K \text { field by joint assimilation of } m_{0} \\
\left.\text { and } m_{1} \text { measurements, with PDEs (Eqs. } 7,8\right) \\
\text { as forecast model. }\end{array}$ & $\mathbf{X}_{\mathbf{f}}^{Y}=\left[\mathbf{Y} ; \mathbf{M}_{0}^{1} ; \ldots ; \mathbf{M}_{0}^{N_{\mathrm{p}}} ; \mathbf{M}_{1}^{1} ; \ldots ; \mathbf{M}_{1}^{N_{\mathrm{p}}}\right]$ \\
\hline $\mathrm{D}$ & $\begin{array}{l}\text { Estimate } S_{\mathrm{S}} \text { field by assimilating } m_{1} \text { measure- } \\
\text { ments only, with PDE (Eq. } 8 \text { ) as forecast model, } \\
\text { in which the } Y \text { forecast ensemble and its corre- } \\
\text { sponding } m_{0} \text { forecast ensemble (obtained from } \\
\text { PDE Eq. 7) are used. }\end{array}$ & $\mathbf{X}_{\mathrm{f}}^{Z}=\left[\mathbf{Z} ; \mathbf{M}_{1}^{1} ; \ldots ; \mathbf{M}_{1}^{N_{\mathrm{p}}}\right]$ \\
\hline $\mathrm{E}$ & $\begin{array}{l}\text { Estimate } S_{\mathrm{S}} \text { field by assimilating } m_{1} \text { measure- } \\
\text { ments only, with PDE (Eq. } 8 \text { ) as forecast model, } \\
\text { in which the posterior mean of } Y \text {, as estimated } \\
\text { in } \mathbf{A} \text {, and its corresponding } m_{0} \text { distribution (ob- } \\
\text { tained from PDE Eq. } 7 \text { ) are used. }\end{array}$ & $\mathbf{X}_{\mathrm{f}}^{Z}=\left[\mathbf{Z} ; \mathbf{M}_{1}^{1} ; \ldots ; \mathbf{M}_{1}^{N_{\mathrm{p}}}\right]$ \\
\hline
\end{tabular}

matrix $\mathbf{K} \in R^{\left(N_{\mathrm{p}}+1\right) n \times N_{\mathrm{p}} N_{\mathrm{o}}}$ is called "Kalman gain", and is computed as

$\mathbf{K}=\mathbf{P}_{\mathrm{f}}^{Y} \cdot \mathbf{H}^{T} \cdot\left(\mathbf{H} \cdot \mathbf{P}_{\mathrm{f}}^{Y} \cdot \mathbf{H}^{T}+\mathbf{R}\right)^{-1}$.

In the context of parameter estimation, we are interested exclusively in updated parameters. Consequently, the ensemble of log-transformed hydraulic conductivity fields is extracted from the updated state-parameter matrix (Eq. 11) as $\mathbf{Y}_{\mathrm{u}}=\mathbf{X}_{\mathrm{u}}^{Y}\left(1: n, 1: N_{\text {ens }}\right)$. The posterior ensemble mean of the hydraulic conductivity is thus computed as $\hat{\boldsymbol{Y}}=\mathbf{Y}_{\mathrm{u}} \cdot \hat{\mathbf{1}}_{N_{\mathrm{ens}}}$, where $\hat{\mathbf{1}}_{N_{\mathrm{ens}}}$ is a $N_{\mathrm{ens}} \times 1$ vector in which all elements are equal to $1 / N_{\text {ens }}$.

A procedure similar to that described above to obtain the ensemble $\mathbf{Y}_{\mathrm{u}}$ by assimilating the zeroth moment of the IRF computed from hydraulic head measurements (Eq. 3) may be devised to derive the specific elastic storage ensemble $\mathbf{Z}_{\mathrm{u}}$, using observations of the first moment of the IRF (Eq. 4). The formulations $\mathrm{D}$ and $\mathrm{E}$, presented in Table 1, provide two possible methods for assembling the forecast matrix in order to estimate the $Z$ field. Since the first temporal moment $m_{1}$ (Eq. 3) depends on the zeroth temporal moment $m_{0}$, as well as the $K$ and $S_{\mathrm{s}}$ fields, the uncertainty on $K$ might affect the estimation of $S_{\mathrm{s}}$. To reduce the influence of the uncertainty on $K$ on the estimation of $S_{\mathrm{s}}$, it is possible, for example, to use the posterior ensemble mean $\hat{\boldsymbol{Y}}$ to solve Eqs. (7) and (8). This assimilation strategy is denoted as $\mathrm{E}$ in Table 1 . In this case, the forecast matrix is expressed as $\mathbf{X}_{\mathrm{f}}^{Z}=\left[\mathbf{Z}, \mathbf{M}_{1}^{1}, \ldots, \mathbf{M}_{1}^{N_{\mathrm{p}}}\right]^{T}$, where $\mathbf{M}_{1}^{i}$ represents the first-moment ensembles for the $i$ th pumping test.
$\mathbf{X}_{\mathrm{f}}^{Z}$ is updated by assimilating the observations of the hydraulic head first-moment $\boldsymbol{d}_{1}=\left[m_{1, i, j}\right] \in R^{N_{\mathrm{p}} N_{0} \times 1}$, where $i$ is the pumping test index and $j$ is the observation well index - and applying equations similar to Eqs. (11) and (12). The ensemble mean of the updated $Z$ is thus computed as $\hat{\boldsymbol{Z}}=\mathbf{Z}_{\mathrm{u}} \mathbf{1}_{N_{\text {ens }}}$. This mean represents the best unbiased estimate of the unknown true parameter. In Sect. 4.1 we show that this approach significantly improves the estimation of $S_{\mathrm{S}}$.

\subsubsection{Parameter estimation by decentralized fusion}

For conciseness, this section describes the DF algorithm to estimate the $K$ field only. The estimation of the $S_{\mathrm{s}}$ field is achieved by applying an analogous procedure.

In the DF approach (Fig. 1b), the data from each pumping test are assimilated separately using a "local" EnKF. The application of the EnKF to each of the $N_{\mathrm{p}}$ pumping tests produces multiple estimates of the hydraulic properties of the aquifer, which are characterized by the means of the posterior ensembles, $\hat{\boldsymbol{Y}}_{\mathrm{u}}^{1}, \ldots, \hat{\boldsymbol{Y}}_{\mathrm{u}}^{N_{\mathrm{p}}}$, and their corresponding posterior covariances, $\mathbf{P}_{\mathrm{u}}^{Y, 1}, \ldots, \mathbf{P}_{\mathrm{u}}^{Y, N_{\mathrm{p}}}$. The objective of the DF algorithm is to merge these estimates and produce an integrated global estimate $\tilde{\mathbf{Y}}$ of the parameters. The multiple estimates are fused using the GMF (Bar-Shalom and Campo, 1986; Shin et al., 2006): 
$\tilde{\boldsymbol{Y}}=\mathbf{W}^{T} \cdot \hat{\boldsymbol{Y}}_{\mathrm{u}}^{1: N_{\mathrm{p}}}=\sum_{i=1}^{N_{\mathrm{p}}} \mathbf{w}_{i} \hat{\boldsymbol{Y}}_{\mathrm{u}}^{i}$,

where the matrix $\mathbf{W}=\left[\mathbf{w}_{1}, \mathbf{w}_{2}, \ldots, \mathbf{w}_{N_{P}}\right]^{T}$, of size $n N_{P} \times n$, includes the $n \times n$ weight matrices $\mathbf{w}_{i}$ $\left(i=1,2, \ldots, N_{\mathrm{p}}\right)$ and the $N_{\mathrm{p}} n \times 1$ vector $\hat{\boldsymbol{Y}}_{\mathrm{u}}^{1: N_{\mathrm{p}}}$ is assembled by vertical concatenation of the means of the posterior ensembles $\hat{\boldsymbol{Y}}_{\mathrm{u}}^{1}, \ldots, \hat{\boldsymbol{Y}}_{\mathrm{u}}^{N_{\mathrm{p}}}$.

The weight matrices in Eq. (14) are given by the solution of the optimization problem:

$\mathbf{W}=\min _{W}|\boldsymbol{Y}-\tilde{\boldsymbol{Y}}|_{2}$,

where $\|\cdot\|_{2}$ represents the Euclidean norm operator. In addition, Eq. (15) is subject to a constraint required to obtain a "best linear unbiased estimate" (BLUE) of $Y$, which is expressed by the following set of linear equations:

$\mathbf{I}_{n, N_{\mathrm{p}}} \cdot \mathbf{W}=\mathbf{I}_{n}$,

where $\mathbf{I}_{n}$ is the $n \times n$ identity matrix, and $\mathbf{I}_{n, N_{\mathrm{p}}}$ is the $n \times n N_{\text {p }}$ matrix formed by horizontal concatenation of $\mathbf{I}_{n}$ for $N_{\mathrm{p}}$ times.

The solution to Eq. (15) is obtained by least-square minimization, which, together with Eq. (16), yields the following linear sets of equations:

$\mathbf{C} \cdot \mathbf{W}=\mathbf{B}$,

where

$\mathbf{C}=\left[\begin{array}{ccc}\mathbf{c}_{1,1} & \ldots & \mathbf{c}_{1, N_{\mathrm{p}}} \\ & \vdots & \\ \mathbf{c}_{N_{\mathrm{p}}-1,1} & \ldots & \mathbf{c}_{N_{\mathrm{p}}-1, N_{\mathrm{p}}} \\ \mathbf{I}_{n} & \ldots & \mathbf{I}_{n}\end{array}\right]$,

$\mathbf{B}=\left[\begin{array}{c}\mathbf{0}_{n} \\ \vdots \\ \mathbf{0}_{n} \\ \mathbf{I}_{n}\end{array}\right]$.

Matrix $\mathbf{C}$ has size $n N_{\mathrm{p}} \times n N_{\mathrm{p}}$, whereas matrix $\mathbf{B}$ has size $n N_{\mathrm{p}} \times n$. In matrix $\mathbf{B}$, Eq. (18a), $\mathbf{0}_{1}$ is a zero matrix with size $n \times n$. The generic term $\mathbf{c}_{i, j}$ in matrix $\mathbf{C}$, Eq. (18a), is given by

$\mathbf{c}_{i, j}=\mathbf{P}_{\mathrm{u}}^{Y, i, j}-\mathbf{P}_{\mathrm{u}}^{Y, i, N_{\mathrm{p}}}$,

where $\mathbf{P}_{\mathrm{u}}^{Y, i, j}$ is the updated cross-covariance matrix for the $Y$ fields estimated from the assimilation of data corresponding to pumping tests $i$ and $j$, which is calculated as

$\mathbf{P}_{\mathrm{u}}^{Y, i, j}=\left(\mathbf{Y}_{\mathrm{u}}^{i}-\hat{\mathbf{Y}}_{\mathrm{u}}^{i}\right)\left(\mathbf{Y}_{\mathrm{u}}^{j}-\hat{\mathbf{Y}}_{\mathrm{u}}^{j}\right)^{T} /\left(N_{\mathrm{ens}}-1\right)$.

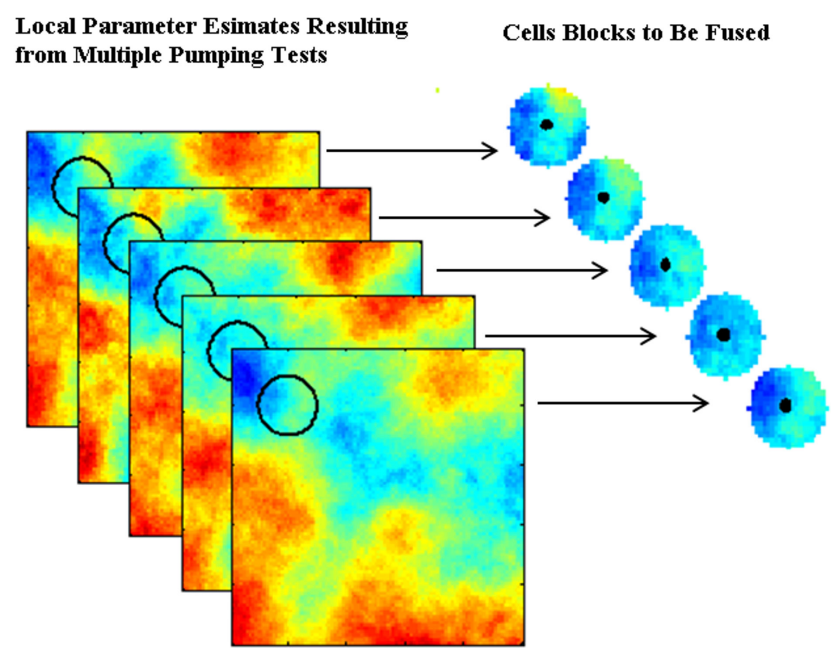

Figure 2. Illustration of localized decentralized fusion. The figure shows five images of hydraulic parameters to be merged. The cells at the center of the circles are the cells to be fused using cell blocks within a specified distance from the center.

From Eq. (17), $\mathbf{W}$ is obtained as

$\mathbf{W}=\mathbf{C}^{-1} \cdot \mathbf{B}$.

Once the weight matrix $\mathbf{W}$ is calculated, it is substituted in Eq. (14) to provide the estimate $\tilde{\boldsymbol{Y}}$. The posterior covariance of $\tilde{\boldsymbol{Y}}$ can be computed as (Shin et al., 2006)

$\tilde{\mathbf{P}}=\mathbf{W}^{T} \cdot \mathbf{P} \cdot \mathbf{W}$

where $\mathbf{P}$ is a $n N_{\mathrm{p}} \times n N_{\mathrm{p}}$ matrix formed by the covariance matrices $\mathbf{P}_{\mathrm{u}}^{Y, i, j}\left(i, j=1, \ldots, N_{\mathrm{p}}\right)$.

\subsubsection{Localization of decentralized fusion}

The inversion of the matrix $\mathbf{C}$ in Eq. (21) constitutes the most intensive part of the GMF. In HT, it is typically required to estimate hydrogeological parameters at high resolution, which often renders the GMF approach computationally very intensive. To circumvent this obstacle, we propose the following novel localized fusion algorithm.

In essence, instead of computing Eq. (21) for all the cells in the domain at once, the fused estimate at any given cell is computed by considering only a circular block of cells within a specified radius around the cell of interest (Fig. 2). The localized DF algorithm visits each cell within the domain sequentially or in parallel and fuses these circular blocks. The resulting fused estimate for the cell at the center is returned, and the algorithm moves to the next cell. Indicating as $n^{\prime}$ $(<n)$ the number of grid cells within a specified distance from the cell of interest, the resulting size for the "local" matrices in Eq. (21) is $n^{\prime} \times n^{\prime} N_{\mathrm{p}}$ for $\mathbf{B}$, and $n^{\prime} N_{\mathrm{p}} \times n^{\prime} N_{\mathrm{p}}$ for $\mathbf{C}$.

The implicit assumption behind this method is that neighboring cells will have the majority of influence on the 
Table 2. Model setting for the numerical experiments.

\begin{tabular}{|c|c|}
\hline \multicolumn{2}{|c|}{ Finite-difference grid properties } \\
\hline $\begin{array}{l}\text { Domain dimensions }[x, y, z](\mathrm{m}, \mathrm{m}, \mathrm{m}) \\
\text { Cell size }[x, y, z](\mathrm{m}, \mathrm{m}, \mathrm{m}) \\
\text { Total number of cells }\end{array}$ & $\begin{array}{l}{[1000,1000,10]} \\
{[10,10,10]} \\
10000\end{array}$ \\
\hline \multicolumn{2}{|l|}{ Boundary conditions } \\
\hline $\begin{array}{l}\text { Dirichlet boundary conditions at } \\
x=0 \mathrm{~m} \\
x=1000 \mathrm{~m} \\
\text { Neumann boundary conditions at } \\
y=0 \mathrm{~m} \\
y=1000 \mathrm{~m}\end{array}$ & $\begin{array}{l}h=45 \mathrm{~m} \\
h=45 \mathrm{~m} \\
\text { no-flow } \\
\text { no-flow }\end{array}$ \\
\hline \multicolumn{2}{|l|}{ Geostatistical parameters } \\
\hline $\begin{array}{l}{\left[\mu_{Y}, \sigma_{Y}, \lambda_{Y}\right]\left(\ln \mathrm{m} \mathrm{day}^{-1}, \ln \mathrm{m} \mathrm{day}^{-1}, \mathrm{~m}\right)} \\
{\left[\mu_{Z}, \sigma_{Z}, \lambda_{Z}\right]\left(\ln \mathrm{m}^{-1}, \ln \mathrm{m}^{-1}, \mathrm{~m}\right)}\end{array}$ & $\begin{array}{l}{[1.5,1,350]} \\
{[-10,1,350]}\end{array}$ \\
\hline \multicolumn{2}{|l|}{ Pumping tests } \\
\hline $\begin{array}{l}\text { Well \#1 }[x, y ; Q]\left(\mathrm{m}, \mathrm{m} ; \mathrm{m}^{3} \mathrm{day}^{-1}\right) \\
\text { Well \#2 }[x, y ; Q]\left(\mathrm{m}, \mathrm{m} ; \mathrm{m}^{3} \mathrm{day}^{-1}\right) \\
\text { Well \#3 }[x, y ; Q]\left(\mathrm{m}, \mathrm{m} ; \mathrm{m}^{3} \mathrm{day}^{-1}\right) \\
\text { Well \#4 }[x, y ; Q]\left(\mathrm{m}, \mathrm{m} ; \mathrm{m}^{3} \mathrm{day}^{-1}\right) \\
\text { Well \#5 }[x, y ; Q]\left(\mathrm{m}, \mathrm{m} ; \mathrm{m}^{3} \mathrm{day}^{-1}\right)\end{array}$ & $\begin{array}{l}{[500,500 ; 500]} \\
{[200,500 ; 500]} \\
{[800,500 ; 500]} \\
{[500,200 ; 500]} \\
{[500,800 ; 500]}\end{array}$ \\
\hline Observation wells & See layout in Fig. 3 \\
\hline
\end{tabular}

estimation. The GMF localization is meant to improve the computational efficiency in two ways: first, the inversion of matrices $\mathbf{C}$ of smaller size is less CPU intensive; second, the fusion algorithm can be directly parallelized on multicore processors.

\subsection{Options for data fusion formulation}

The forecast matrix $\mathbf{X}_{f}$ can be assembled according to different formulations of the data fusion problem. Table 1 shows a list of the formulations investigated herein.

Formulations A, B, and C seek the estimation of the $Y$ field. Formulation A consists of assimilating measurements of the zeroth temporal moment $m_{0}$ (Eq. 3), with the forecast model given by numerical solution of the PDE (Eq. 7). Formulation B consists of assimilating measurements of the first temporal moment $m_{1}$ (Eq. 4), with the forecast model given by numerical solution of the PDE (Eq. 8), in which the $K$ forecast ensemble and its corresponding $m_{0}$ forecast ensemble, in turn obtained from the numerical solution of the PDE (Eq. 7), are used. In formulation C, measurements of both $m_{0}$ and $m_{1}$ are assimilated, and the forecast model is obtained by solving Eqs. (7) and (8) combined.

Formulations $\mathrm{D}$ and $\mathrm{E}$ aim at estimating the $Z$ field. In the estimation of $S_{\mathrm{s}}$, it is possible to find a one-to-one correspondence between $S_{\mathrm{S}}$ and $m_{1}$ based on Eq. (8) if the $K$ field and its corresponding $m_{0}$ field are known. However, since the $K$ field is unknown, one can choose instead to represent

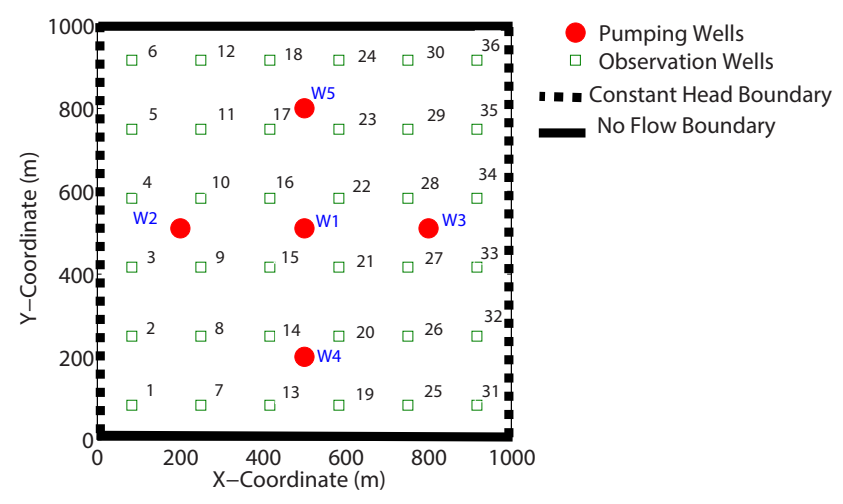

Figure 3. Locations of pumping wells, observation wells, and boundary conditions.

it using, for example, its forecast ensemble $\mathbf{Y}$, or a best unbiased estimate, calculated as the mean of the posterior ensemble $\hat{\boldsymbol{Y}}$ obtained in formulation A. These alternatives are investigated in formulations $\mathrm{D}$ and $\mathrm{E}$. In both instances, measurements of $m_{1}$ are assimilated, and the forecast model consists of the numerical solution of the PDE (Eq. 8). In formulation $\mathrm{D}$, the $K$ forecast ensemble and its corresponding $m_{0}$ forecast ensemble, obtained from the numerical solution of the PDE (Eq. 7), are used. Instead, in formulations E, the posterior mean of $Y$, as estimated in formulation A, and its corresponding $m_{0}$ distribution, obtained from the numerical solution the PDE (Eq. 7), are used.

\section{Numerical experiments}

\subsection{Model setup}

The testing of the inversion schemes proposed in this work is based on a number of hypothetical two-dimensional cases. The method is, however, directly applicable to threedimensional problems. We consider a two-dimensional horizontal $1 \mathrm{~km} \times 1 \mathrm{~km}, 10 \mathrm{~m}$ thick confined aquifer, discretized into 10000 cells (100 gridblocks along the $x-y$ coordinate directions, and a single grid block along the $z$ direction). Table 2 and Fig. 3 provide detailed descriptions of data regarding the aquifer model.

The aquifer is subject to constant-head boundary conditions on the left and right edges of the domain, at which the hydraulic head $h$ is set equal to $45 \mathrm{~m}$. Anywhere else no-flow boundary conditions are imposed. The "true" $K$ and $S_{\mathrm{s}}$ fields in the aquifer are assumed to fit to the geostatistical models introduced in Sect. 2.3.1 and generated synthetically using the sequential Gaussian simulation algorithm SGSIM (Deutsch and Journel, 1997), with the geostatistical parameters $\mu_{Y}=1.5 \mathrm{ln}$ $\mathrm{m} \mathrm{day}^{-1}, \sigma_{Y}=1 \ln \mathrm{mday}^{-1}, \lambda_{Y}=350 \mathrm{~m}, \mu_{Z}=-10 \ln \mathrm{m}^{-1}$,

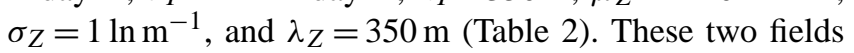
are used in five MODFLOW-2000 (Harbaugh et al., 2000) 
simulations to reproduce the aquifer response to five separate pumping tests, conducted from the locations and with the pumping rates specified in Table 2 and Fig. 3.

The duration of these hypothetical pumping tests is 10 days. The output of each simulation provides the reference system from which the collection of hydraulic head data is simulated. Hydraulic head observations are recorded from a network of 36 monitoring wells, whose locations are depicted in Fig. 3.

Three sets of numerical experiments are carried out to evaluate and compare the performances of the $\mathrm{CF}$ and $\mathrm{DF}$ schemes. The first experiment set investigates the performance of different formulations of the forecast matrix, as listed in Table 1, using the CF approach. The second experiment set is similar to the first experiment set, but the DF approach is used instead. In the third experiment, we investigate the effects of assimilating temporal moments instead of hydraulic head data. To do this, we compare the $Y$ and $Z$ fields obtained by direct assimilation of transient hydraulic head data with those obtained by assimilating zeroth and first temporal moments of the IRF. The comparison in the third experiment set is limited only to a single pumping test at well number 1 in Fig. 3. In all experiment sets, the parameters characterizing the geostatistical models of $Y$ and $Z$ are assumed to be known as prior information and equal to those of the "true" fields given in Table 2. The prior ensembles of $Y$ and $Z$ realizations are assumed be uncorrelated.

In the three experiment sets, the size $N_{\text {ens }}$ of the ensemble is 200 . The temporal moments at each observation well are estimated using Eqs. (3) and (4). Since the temporal moments are assumed to be the measured quantities, their measurement error is assumed to fit to a normal distribution with zero mean and standard deviation equal to the corresponding forecast's standard deviation multiplied by 0.01 .

\subsection{Performance metrics}

The performances of the fusion methods may be evaluated qualitatively by visual comparison of the maps of the estimated hydraulic parameters, represented by the average distributions $\hat{\boldsymbol{Y}}$ and $\hat{\boldsymbol{Z}}$ (Sect. 2.3.2), with the corresponding maps of the "true" reference fields. In addition, a quantitative evaluation of these performances is achieved using the following statistics: the mean absolute error $L_{1}$, the root mean square error $L_{2}$, the mean error $\mu_{\mathrm{e}}$, and the correlation coefficient $r$. $L_{1}$ is computed as

$L_{1}=\frac{1}{n} \sum_{i=1}^{n}\left|\phi_{\text {true }}(i)-\hat{\phi}(i)\right|$,

where $\phi \operatorname{true}(i)$ is the value of "true" parameter at the grid cell $i$ and $\hat{\phi}(i)$ is the corresponding value of estimated parameter. $L_{2}$ is computed as
$L_{2}=\sqrt{\frac{1}{n} \sum_{i=1}^{n}\left[\phi_{\text {true }}(i)-\hat{\phi}(i)\right]^{2}}$.

The correlation between the estimated parameter field and the true parameter field, both represented as two-dimensional images, can be computed using Pearson's correlation coefficient $r$ as follows:

$$
r=\frac{\sum_{i=1}^{n_{\mathrm{r}}} \sum_{j=1}^{n_{\mathrm{c}}}\left[\phi_{\text {true }}(i, j)-\bar{\phi}_{\text {true }}\right][\hat{\phi}(i, j)-\bar{\phi}]}{\sqrt{\sum_{i=1}^{n_{\mathrm{r}}} \sum_{j=1}^{n_{\mathrm{c}}}\left[\phi_{\text {true }}(i, j)-\bar{\phi}_{\text {true }}\right]^{2} \sum_{i=1}^{n_{\mathrm{r}}} \sum_{j=1}^{n_{\mathrm{c}}}[\hat{\phi}(i, j)-\bar{\phi}]^{2}}},
$$

where $\bar{\phi}_{\text {true }}$ and $\bar{\phi}$ are the overall means of the true and the estimated parameter fields, respectively, and $n_{\mathrm{r}}$ and $r_{\mathrm{c}}$ are the number of rows and the number of columns of the twodimensional field, respectively. Values of $r$ range between 1 and -1 , with $r=1$ indicating perfect positive linear correlation, $r=0$ indicating no correlation, and $r=-1$ indicating perfect negative correlation. Finally, the error mean $\mu_{\mathrm{e}}$ is obtained as

$\mu_{\mathrm{e}}=\frac{1}{n} \sum_{i=1}^{n}\left[\phi_{\text {true }}(i)-\hat{\phi}(i)\right]$

and is meant to provide a measure of the biasedness of the estimate. Values of $\mu_{\mathrm{e}}$ close to zero indicate an unbiased estimate.

\section{Results and discussions}

\subsection{Centralized fusion of HT data}

In this section, the performance of each of the forecast formulations given in Table 1 is evaluated using the CF scheme (Fig. 1). The results of the inversion tests are summarized in Table 3, which reports values of the four performance statistics, $L_{1}, L_{2}, r$ and $\mu_{\mathrm{e}}$ (Eqs. 23-26) for the formulation schemes A-E. As explained in Sect. 2.4, formulations A-C seek the estimation of the $Y$ field. The comparison of the metrics $L_{1}, L_{2}$, and $r$ reported in Table 4 reveals that the $\mathrm{CF}$ scheme performs significantly better under formulation $\mathrm{A}$ than under formulation C. In turn, formulation B is slightly less effective than formulation $\mathrm{C}$.

These results find an explanation in that with formulation A the $Y$ field is estimated by assimilating $m_{0}$ data only, whereas with formulation $\mathrm{C}$ the $Y$ field is estimated by assimilating both $m_{0}$ and $m_{1}$ data. While in formulation $A$ the heterogeneity of the $Y$ field affects directly the spatial variability of $m_{0}$ via the PDE (Eq. 7), in formulation C such heterogeneity influences both the $m_{0}$ and $m_{1}$ spatial distributions via PDEs (Eqs. 7, 8). In addition, the spatial variability of $m_{1}$ depends not only on $Y$ but also on $Z$. This makes the 
(a) True $Y$

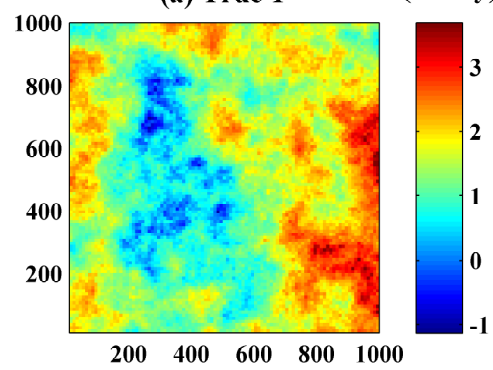

(c) Estimated $Y$

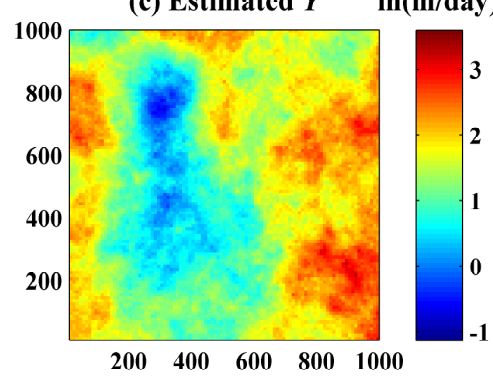

(e) Estimated vs. True $Y$ $\ln (\mathbf{m} /$ day $)$

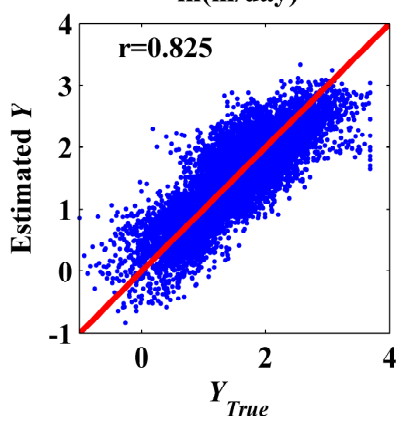

(b) True $Z$

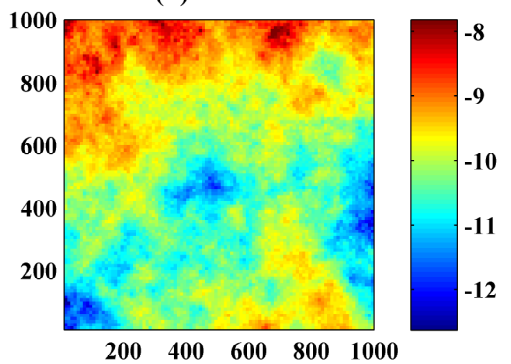

(d) Estimated $Z$

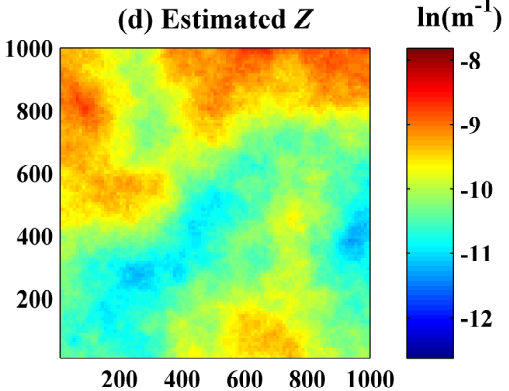

(f) Estimated vs. True $Z$ $\ln \left(\mathbf{m}^{-1}\right)$

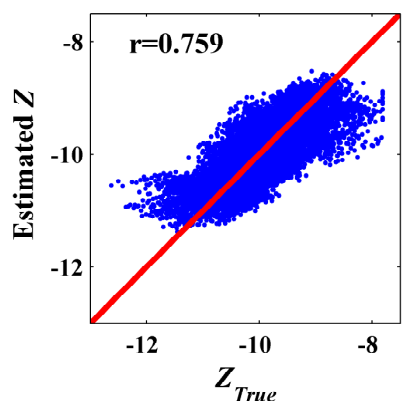

Figure 4. Maps of (a, b) the "true" reference $Y$ and $Z$ fields, and (c, d) the $Y$ and $Z$ fields estimated using the CF scheme with formulations A and E, respectively. (e, f) Scatter plots of $\boldsymbol{Y}_{\text {true }}$ vs. $\hat{\boldsymbol{Y}}$ and $\boldsymbol{Z}_{\text {true }}$ vs. $\hat{\boldsymbol{Z}}$.

estimation of $Y$ using PDEs (Eqs. 7, 8) less effective given the added uncertainty in $Z$.

In the case of formulation $\mathrm{B}$, the performance of the $\mathrm{CF}$ scheme is even lower than with formulation C since only $m_{1}$ data are assimilated and thus the impact of the added uncertainty in $Z$ is inevitably more pronounced. In Table 3, it is worth observing that for all formulations $\mathrm{A}-\mathrm{C}$, the mean error $\mu_{\mathrm{e}}$ is very low, on the order of $10^{-5}$, which provides substantial evidence of the unbiasedness of the estimates obtained by $\mathrm{CF}$.

Figure $4 \mathrm{a}$ and c present the maps of the "true" reference field $\boldsymbol{Y}_{\text {true }}$ and the average of the update ensemble $\hat{\boldsymbol{Y}}$ obtained using the forecast formulation A, respectively. The similarity between the two maps is remarkable. Figure 4e shows a scatter plot obtained using the components of $\boldsymbol{Y}_{\text {true }}$ on the $x$ axis, and the corresponding components of $\hat{\boldsymbol{Y}}$ on the $y$ axis. The data points in this plot tend to gather along the identity line, which provides a further visual proof of the satisfactory performance of the $\mathrm{CF}$ scheme.

In formulations D and E (Table 1), the estimation of the $Z$ field is sought using the CF approach. The values of the metrics $L_{1}, L_{2}$, and $r$ given in Table 3 indicate that with formulation $\mathrm{D}$ the $\mathrm{CF}$ scheme performs significantly worse than with formulation $\mathrm{E}$. Indeed, estimating the $Z$ field based exclusively on $m_{1}$ data through the PDE (Eq. 8) is inevitably affected by the uncertainty on the $Y$ and the $m_{0}$ fields, in a fashion very similar to that highlighted above for formulation B. A similar outcome has been observed by other researchers (Yin and Illman, 2009). It is interesting to note that formulations B and D are substantially the same, although they attempt to estimate different parameters. Thus it is not coincidental that their performance exhibits the two lowest estimations. 
Table 3. Performance statistics for the formulations of Table 1 using CF.

\begin{tabular}{|c|c|c|c|c|c|}
\hline \multirow[t]{3}{*}{ Performance statistics } & \multicolumn{5}{|c|}{ Formulation } \\
\hline & \multicolumn{3}{|c|}{$Y=\ln K$} & \multicolumn{2}{|c|}{$Z=\ln S_{\mathrm{s}}$} \\
\hline & A & $\mathrm{B}$ & $\mathrm{C}$ & $\mathrm{D}$ & $\mathrm{E}$ \\
\hline Mean absolute error: $L_{1}$ & 0.318 & 0.353 & 0.343 & 0.596 & 0.363 \\
\hline Root mean square error: $L_{2}$ & 0.408 & 0.446 & 0.438 & 0.730 & 0.460 \\
\hline Correlation coefficient: $r$ & 0.825 & 0.787 & 0.803 & 0.292 & 0.759 \\
\hline Mean error: $\mu_{\mathrm{e}}$ & $1.40 \times 10^{-5}$ & $1.54 \times 10^{-5}$ & $1.01 \times 10^{-5}$ & $-6.13 \times 10^{-6}$ & $-5.31 \times 10^{-6}$ \\
\hline
\end{tabular}

Table 4. Performance statistics for the formulations of Table 1 using DF.

\begin{tabular}{|c|c|c|c|c|c|}
\hline \multirow[t]{3}{*}{ Performance statistics } & \multicolumn{5}{|c|}{ Formulation } \\
\hline & \multicolumn{3}{|c|}{$Y=\ln K$} & \multicolumn{2}{|c|}{$Z=\ln S_{\mathrm{s}}$} \\
\hline & A & B & $\mathrm{C}$ & $\mathrm{D}$ & $\mathrm{E}$ \\
\hline Mean absolute error: $L_{1}$ & 0.412 & 0.458 & 0.442 & 0.776 & 0.466 \\
\hline Root mean square error: $L_{2}$ & 0.521 & 0.570 & 0.556 & 0.953 & 0.605 \\
\hline Correlation coefficient: $r$ & 0.723 & 0.683 & 0.700 & 0.246 & 0.645 \\
\hline Mean error: $\mu_{\mathrm{e}}$ & $2.0 \times 10^{-2}$ & $2.2 \times 10^{-2}$ & $1.4 \times 10^{-2}$ & $-1.05 \times 10^{-1}$ & $-9.1 \times 10^{-2}$ \\
\hline
\end{tabular}

Based on the results of formulation $\mathrm{B}$, the estimation of $Z$ may be improved if the uncertainty on the $Y$ and $m_{0}$ fields can be reduced. Formulation E (Table 1) stems from the idea of using the best unbiased estimate $\hat{\mathbf{Y}}$ obtained with formulation $\mathrm{A}$, and the corresponding $m_{0}$ field calculated by solving the PDE (Eq. 7), within the the forecast model based on the PDE (Eq. 8) and assimilated $m_{1}$ measurements only, as in formulation D. The values of $L_{1}, L_{2}$, and $r$ shown in Table 3 reveal that this solution allows for a significant improvement in the estimation of the $Z$ field, and the performance of the $\mathrm{CF}$ approach becomes comparable with that observed in formulations A-C, when estimating the $Y$ field. Note in Table 3 that with both formulations $\mathrm{D}$ and $\mathrm{E}$ the $\mathrm{CF}$ approach produces negligible values of $\mu_{\mathrm{e}}\left(10^{-6}\right)$, which demonstrates that the estimates of $Z$ are substantially unbiased.

Figure $4 \mathrm{~b}$ and $\mathrm{d}$ depict the "true" $Z$ field and that estimated by $\mathrm{CF}$ using formulation $\mathrm{E}$, respectively. A comparison between the two maps shows that the CF scheme is able to capture fairly well the spatial heterogeneity of $Z$. Figure $4 \mathrm{f}$ shows a scatter plot of $\boldsymbol{Z}_{\text {true }}$ against $\hat{\boldsymbol{Z}}$. Similar to Fig. $4 \mathrm{e}$, the data are distributed along the identity line, that is, a general agreement between "true" and the estimated $Z$ can be observed. However, Fig. 4f shows that higher and lower values of $Z$, located on the "tails" of the distribution, are not well identified, which highlights the tendency of the CF scheme to produce smoothed estimates of the $Z$ field.

Plots in Fig. 5 compare the simulated heads using the estimated $Y$ and $Z$ fields using CF methods with heads obtained by simulating true parameter fields. Figure 5a shows a scatter plot of simulated heads versus reference heads resulting from the five pumping tests and for heads observed at
36 observation wells. The performance statistics $L_{1}, L_{2}$, and $r$ are $0.09,0.015$, and 0.998 , respectively, indicating fairly good performance of the inversion method. Figure $5 \mathrm{~b}-\mathrm{f}$ show one sample of hydraulic head hydrographs resulting from the five pumping tests at observation well 15 (see Fig. 3), which is located approximately in the middle of the simulated domain. The figures show a general agreement between observed and simulated head hydrographs.

In the tests presented above, the average CPU time required to calculate the spatial distributions of temporal moments - that is, to solve either of the PDEs (Eqs. 7, 8) using MODFLOW-2000 (Harbaugh et al., 2000) - is about $2 \mathrm{~s}$ per run. In practice, a forecast simulation with an ensemble size $N_{\text {ens }}$ of 200 requires a CPU time on the order of minutes. This is because the moment-generating PDEs (Eqs. 7, 8) are Poisson-type equations, which are computationally much less intensive to solve than the parabolic PDE (Eq. 5) from which they are derived. In this regard, note the PDE (Eq. 5) is time dependent, whereas in PDEs (Eqs. 7, 8) the time variable is eliminated by integration (Eq. 2).

Considering the temporal moments of the IRF measurements allows also for a significant reduction of the CPU requirements of data assimilation. In the numerical experiments conducted here, 36 observation wells are used to monitor the hydraulic head during each pump test. In each observation well 100 temporal measurements are recorded, resulting in 3600 measurements per single pumping test. Since we assumed that five pumping tests are performed to characterize the aquifer, the total number of available hydraulic head data is $(36 \times 100 \times 5) 18000$. Direct assimilation of transient hydraulic head data using either the EnKF or the 

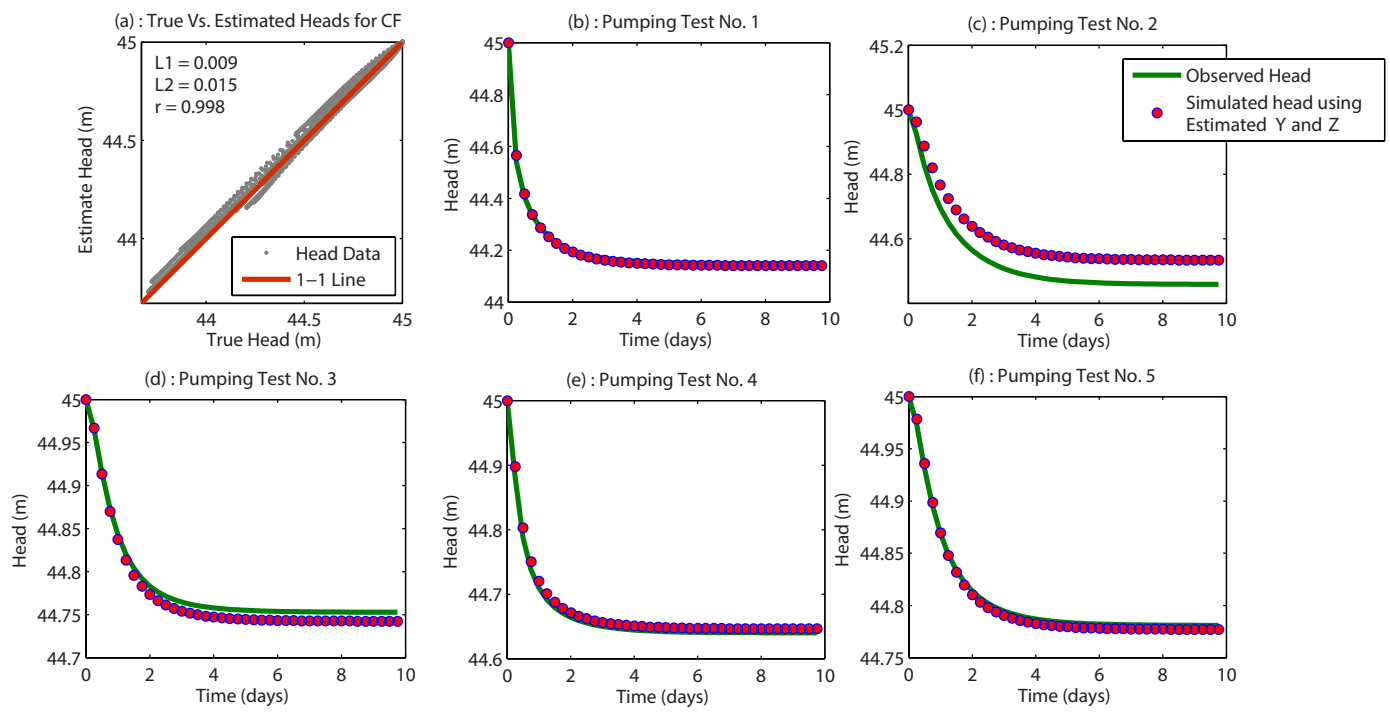

Figure 5. Verification of the CF inversion method by comparing true hydraulic heads and hydraulic heads simulated using estimated parameters. (a) shows the scatter plot of true heads on $x$ axis and estimated heads on the $y$ axis for the five pumping tests. (b-f) show time series for the true and the estimated hydraulic heads at observation well no. 15 in Fig. 3.

ensemble smoother (Evensen, 2009) would require, respectively, the inversion (Eq. 13) of a $180 \times 180$ matrix for each of the 100 measurement times, or the all-at-once inversion of a $18000 \times 18000$ matrix. In either situation, the computational effort would not be trivial. Instead, by introducing temporal moments, for example when estimating the $Y$ field with formulation A, the data assimilation step involves the inversion of a $180 \times 180$ matrix only once.

\subsection{Decentralized fusion of HT data}

In this section, the DF scheme based on the GMF (Sect. 2.3.3) is employed to estimate aquifer parameters based on the same HT data used in the previous section with the $\mathrm{CF}$ scheme. Similar to the set of experiments used to evaluate the CF inversion method, this experiments set investigates formulations A-C to estimate the $Y$ field, and formulations $\mathrm{D}$ and $\mathrm{E}$ to estimate the $Z$ field. It is worth noting that forecast formulations of the forecast matrix in the DF inversion are slightly different from those used in the CF inversion. Forecast matrices in the DF inversion are formulated for each local inversion, while in the CF inversion a single global forecast matrix is formulated as shown in Table 1.

Following the approach outlined in Sect. 2.3.3 to reduce computational intensity, in the calculation of the weight coefficients W (Eq. 21), for each grid cell, only cells within a radius of $50 \mathrm{~m}$ are used in the inversion of the matrix $\mathbf{C}$. The results of preliminary numerical tests (not shown here) have suggested that, in this problem, no significant improvement in accuracy is achieved if this radius is increased beyond $50 \mathrm{~m}$, while a significant increase in computational cost is required.
The performance criteria for the DF method using different formulations are summarized in Table 4. Comparing performance criteria for the DF method shown in Table 4 with performance criteria for the $\mathrm{CF}$ method in Table 3, reveals that the performance of different formulations is independent from the fusion method used. For example, formulation A outperforms formulations $\mathrm{B}$ and $\mathrm{C}$ in estimating the $Y$ field for both $\mathrm{CF}$ and DF methods, and formulation $\mathrm{E}$ outperforms formulation $\mathrm{D}$ in estimating $Z$ for both $\mathrm{CF}$ and $\mathrm{DF}$.

Figure 6 shows the "local" estimates of the $Y$ field obtained using formulation A of the EnKF to assimilate HT data collected separately in each of the five pumping tests. Figure 6f shows the global estimate of the $Y$ field produced by Eq. (14), and Fig. $6 \mathrm{~g}$ show the "true" $Y$ field. The similarity between the two maps in Fig. $6 \mathrm{f}$ and $g$ indicates that the DF scheme is able to estimate fairly well the spatial distribution of hydraulic conductivity. In Fig. 6h, the scatter plot of $\boldsymbol{Y}_{\text {true }}$ vs. $\tilde{\boldsymbol{Y}}$ provides further proof of the good performance of the DF scheme. The resulting correlation coefficient, $r$, between the two distributions is equal to 0.723 , which is less than that obtained by using the CF scheme with formulation A $(r=0.825$, see formulation A in Table 3$)$.

Figure 7 shows the estimations of the $Z$ field obtained using formulation $\mathrm{E}$ and applying the EnKF separately to each of the five pumping tests. Figure $7 \mathrm{f}$ and $\mathrm{g}$ shows the DF global estimate of the $Z$ field and the "true" reference field, respectively. The comparison of the two maps in subpanels $f$ and $g$ indicates that the DF scheme is able to capture the main features of the heterogeneity of the $Z$ field. The scatter plot of $\boldsymbol{Z}_{\text {true }}$ vs. $\tilde{\boldsymbol{Z}}$ shows that the correlation coefficient $r$ is equal to 0.645 , which is smaller than that produced by the 

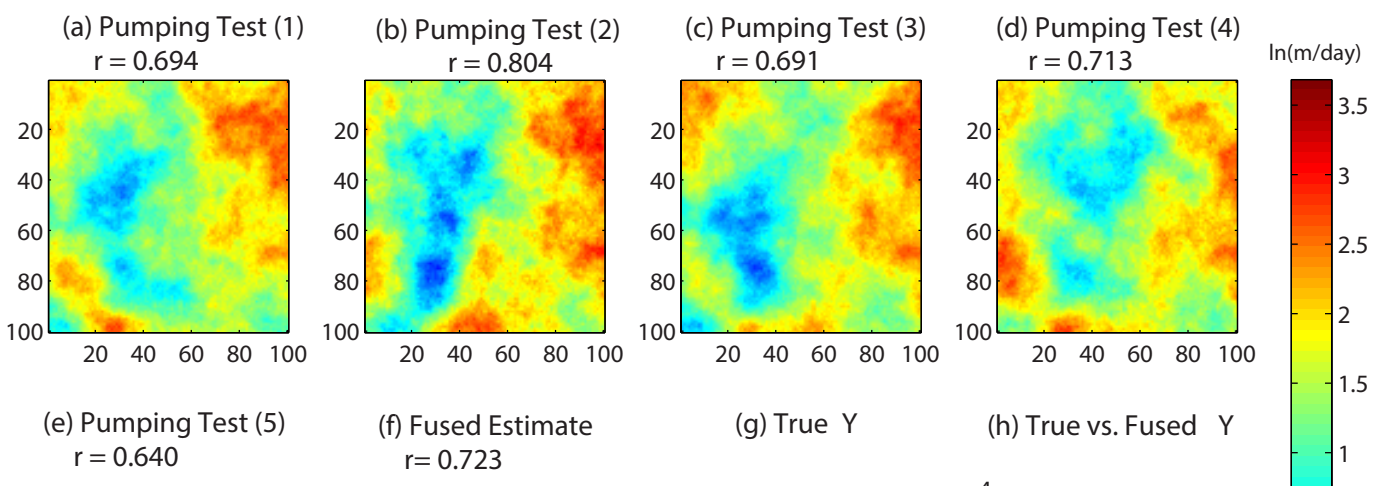

(g) True Y
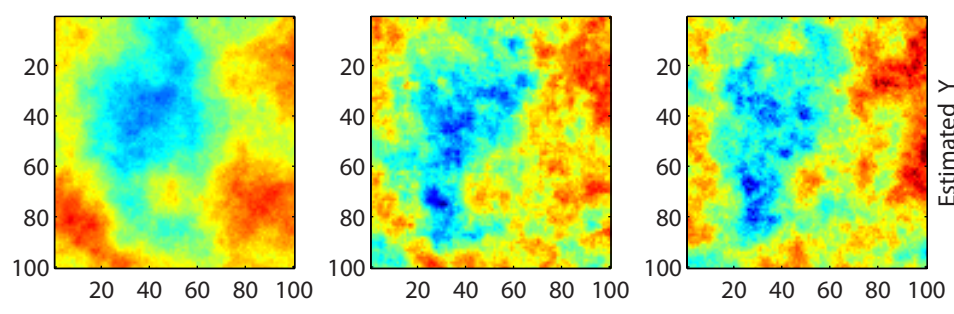

(h) True vs. Fused Y

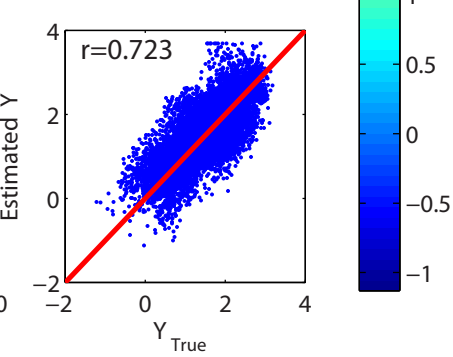

Figure 6. Maps of the $Y$ field obtained with (a-e) local EnKF estimates for each of the five hypothesized pumping tests, and (f) the application of the DF scheme. The "true" reference field is given in (g). (h) shows the scatter plot of $\boldsymbol{Y}_{\text {true }}$ vs. $\tilde{\boldsymbol{Y}}$.
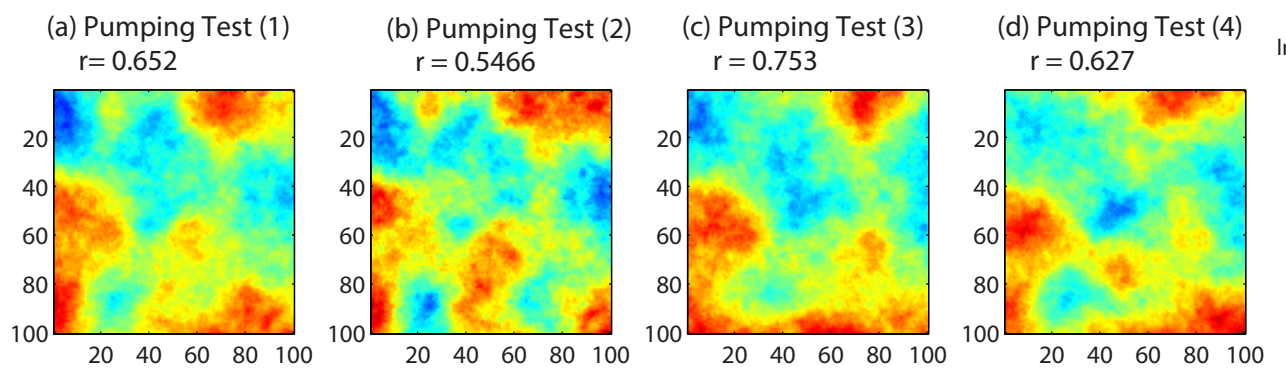

$\ln \left(\mathrm{m}^{-1}\right)$

(e) Pumping Test (5) $r=0.769$
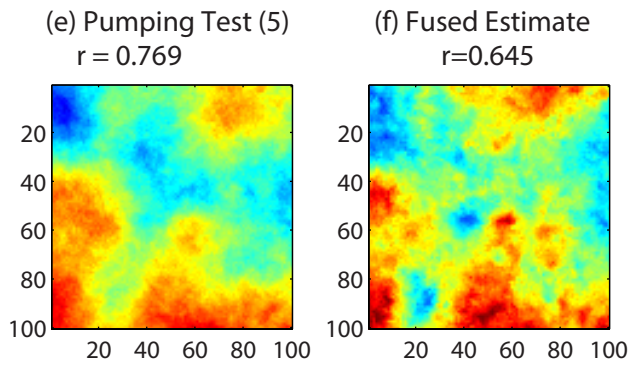

(g) True Z

(h) True Vs. Fused Z
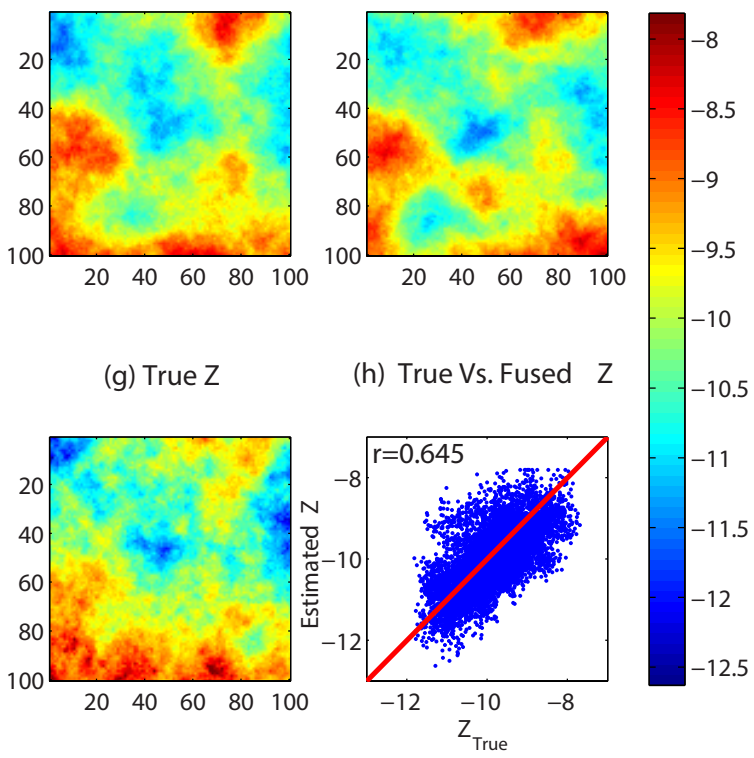

Figure 7. Maps of the $Z$ field obtained with (a-e) local EnKF estimates for each of the five hypothesized pumping tests, and (f) the application of the DF scheme. The "true" reference field is given in (g). (h) shows the scatter plot of $\boldsymbol{Z}_{\text {true }}$ vs. $\tilde{\boldsymbol{Z}}$.

CF scheme with formulation $\mathrm{E}(r=0.759$, see formulation $\mathrm{E}$ in Table 3).

Figure 8 compares hydraulic heads obtained by simulating the estimated $Y$ and $Z$ fields using DF method with observed hydraulic heads. Comparing the performance metrics of the DF method, shown in Fig. 8a, with the performance metrics of the CF method, shown in Fig. 5a, indicates that the performance of CF inversion performs better than that of DF inversion. The hydraulic heads at observation well no. 15 are plotted in Fig. 8b-f for the five pumping tests. A generally fair agreement can be observed between the simulated heads and true ones. 

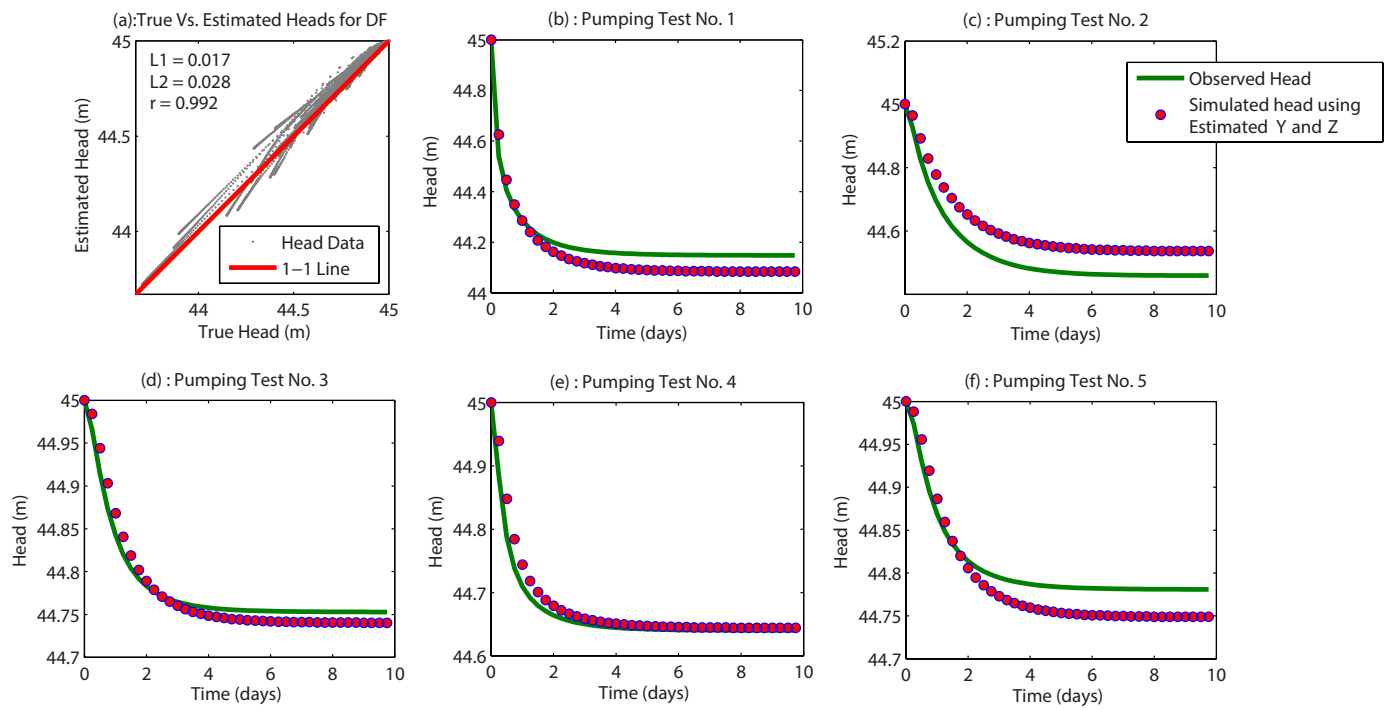

Figure 8. Verification of the DF inversion method by comparing true hydraulic heads and hydraulic heads simulated using estimated parameters. (a) shows the scatter plot of true heads on the $x$ axis and estimated heads on the $y$ axis for the five pumping tests. (b)-(f) show time series for the true and the estimated hydraulic heads at observation well no. 15 in Fig. 3.

The results presented above indicate that, in the joint estimation of $Y$ and $Z$, the $\mathrm{CF}$ scheme consistently outperforms the DF scheme. This can be explained by observing that all of the $r$ coefficients obtained with "local estimations", that is, the application of the EnKF separately to the five pumping tests (panels a-e in Figs. 6 and 7), are smaller than the corresponding coefficients produced by the CF scheme (see formulations $\mathrm{A}$ and $\mathrm{E}$ in Table 3), which applies the EnKF "globally", that is, to the five pumping tests altogether. Since the GMF (Eq. 14) constitutes in essence a weighted average of the "local" estimates of the $Y$ and $Z$ fields, with weights (Eq. 21) that are inversely related to the corresponding "local" covariances (Shin et al., 2006), it produces fused estimates with a coefficient $r$ that cannot be larger than those associated with the best "local" estimate and, consequently, those obtained with the "global" CF estimate. However, the DF scheme has an operational advantage over the CF scheme, in that the "raw" transient data are not required to apply fusion. Indeed, only estimates of the hydraulic parameter field and the covariances are required.

Note also that when applying the DF scheme to the considered problem, the inversion of the matrix $\mathbf{C}$ (Eq. 21) would be computationally overwhelming since its size $\left(n N_{\mathrm{p}} \times n N_{\mathrm{p}}\right)$ is equal to 50000 by 50000 . This application is made possible only by implementing the localized DF described in Sect. 2.3.4. By doing so, the algorithm requires about $40 \mathrm{CPU}$ minutes to complete the calculations without parallelization of the computation. Using a multicore computer would further reduce this time by a factor roughly equal to the number of processors available.
Table 5. Performance statistics for estimates resulting from assimilating transient hydraulic head data and temporal moment data obtained from a single pumping test at well no. 1.

\begin{tabular}{lcccc}
\hline Performance statistics & \multicolumn{4}{c}{ Data assimilated } \\
\cline { 2 - 5 } & Hydraulic head & Temporal moments \\
\cline { 2 - 5 } & $Y$ & $Z$ & $Y$ & $Z$ \\
\hline Mean absolute error: $L_{1}$ & 0.337 & 0.384 & 0.406 & 0.398 \\
Root mean square error: $L_{2}$ & 0.420 & 0.479 & 0.509 & 0.521 \\
Correlation coefficient: $r$ & 0.908 & 0.880 & 0.694 & 0.628 \\
\hline
\end{tabular}

\subsection{Assimilating transient head data versus assimilating temporal moments}

While assimilating temporal moments instead of the transient data itself provides a significant saving in CPU time, it is important to verify to what extent this option affects the accuracy of the estimation. To do so, we conduct an experiment whose goal is to compare the performances of the EnKF when temporal moments are assimilated and when the "raw" transient hydraulic head data are assimilated. In this experiment, we use data from a single pumping test at well no. 1 in Fig. 3. Using data from a single pumping test allows reducing the scale of the data assimilation problem, thereby limiting the associated computational effort, without affecting the generality of conclusions drawn from the experiment.

Table 5 summarizes the performance statistics of the two approaches. One can observe that assimilating the transient data lead to better results compared with assimilating the temporal moments. This observation can be made for the estimation of both $Y$ and $Z$ fields and can be explained by 
information loss resulting from lumping transient head data into low-order temporal moments. However, while assimilating transient head data provides a better characterization than using temporal moments, the associated computational cost is drastically higher. For example, in the case investigated here, the overall CPU time required by the transient data formulation is about 40 times larger than that required by the temporal moment formulation.

It is worth noting that the correlation coefficient $r$ for estimation of $Y$ resulting from a single pumping test $(r=0.908$ in Table 5) is higher than that resulting from five pumping tests ( $r=0.825$ in Table 3$)$, while the $L_{1}$ and $L_{2}$ statistics are better (lower) for multiple pumping tests. This is due to the fact that the correlation coefficient $r$ is invariant with respect to linear transformation of the two fields, and thus $r$ provides a measure of similarity in the structure of spatial variability with no information about the Euclidean distance between the two fields, which is provided by $L_{2}$.

\section{Conclusions}

In this work, two approaches have been developed and implemented to characterize the spatial variability of aquifers' hydraulic properties at high resolution: centralized fusion (CF) and decentralized fusion (DF). CF utilizes a global EnKF (ensemble Kalman filter) scheme to simultaneously invert data obtained from multiple pumping tests. DF uses the generalized Millman formula (GMF) to merge together estimates obtained from "local" EnKF applications to each of the pumping tests. The proposed inversion methods assimilated the zeroth and first temporal moments of the impulse response function (IRF) inferred from hydraulic head data collected in monitoring wells, which significantly expedites the stochastic simulation procedures.

The performance of the fusion schemes, measured as the deviation of the estimated field from the "true" reference field, are promising for both inversion schemes. The numerical tests presented in this work show that the CF scheme using the global EnKF consistently outperforms the DF scheme based on the GMF. To optimize the inversion procedures, different formulations of the forecast matrix were investigated, and results indicate that the estimation of the aquifer parameters is significantly affected by the chosen formulation. For instance, the estimation of the specific elastic storage field was significantly improved by using a specific formulation of the forecast matrix based on the assimilation of measurements of the first temporal moment of the impulse response function, with the posterior mean of hydraulic conductivity obtained with the assimilation of measurements of the zeroth temporal moment.

It is finally important to point out that in the numerical experiments presented here the structure of the geostatistical model and its parameters are assumed be be known a priori. Since most often this hypothesis is not met, it is necessary to extend the methodologies developed in this work to jointly identifying the geostatistical model. This is the subject of an ongoing research effort.

Acknowledgements. We would like to express our great appreciation to Colorado State University Libraries Open Access Research and Scholarship Fund for their the financial support to publish this article. We would like also to thank the three anonymous reviewers for their valuable comments.

Edited by: A. Guadagnini

\section{References}

Bakker, M., Maas, K., and Von-Asmuth, J. R.: Calibration of transient groundwater models using time series analysis and moment matching, Water Resour. Res., 44, W04420, doi:10.1029/2007WR006239, 2008.

Bar-Shalom, Y. and Campo, L.: The effect of the common process noise on the two-sensor fused-track covariance, IEEE T. Aero. Elec. Sys., AES-22, 803-805, doi:10.1109/TAES.1986.310815, 1986.

Berg, S. J. and Illman, W. A.: Three-dimensional transient hydraulic tomography in a highly heterogeneous glaciofluvial aquifer-aquitard system, Water Resour. Res., 47, W10507, doi:10.1029/2011WR010616, 2011.

Berg, S. J. and Illman, W. A.: Field Study of Subsurface Heterogeneity with Steady-State Hydraulic Tomography, Ground Water, 51, 29-40, doi:10.1111/j.1745-6584.2012.00914.x, 2013.

Bohling, G. C. and Butler, J. J.: Inherent limitations of hydraulic tomography, Ground Water, 48, 809-824, doi:10.1111/j.17456584.2010.00757.x, 2010.

Bohling, G. C., Butler, J. J., Zhan, X., and Knoll, M. D.: A field assessment of the value of steady shape hydraulic tomography for characterization of aquifer heterogeneities, Water Resour. Res., 43, W05430, doi:10.1029/2006WR004932, 2007.

Butler Jr., J. J.: The design, performance, and analysis of slug tests, CRC Press, Boca Raton, FL, USA, 1998.

Butler Jr., J. J., McElwee, C. D., and Bohling, G. C.: Pumping tests in networks of multilevel sampling wells: motivation and methodology, Water Resour. Res., 35, 3553, doi:10.1029/1999WR900231, 1999.

Cardiff, M. and Barrash, W.: 3-D transient hydraulic tomography in unconfined aquifers with fast drainage response, Water Resour. Res., 47, W12518, doi:10.1029/2010WR010367, 2011.

Cardiff, M., Barrash, W., and Kitanidis, P. K.: A field proof-ofconcept of aquifer imaging using 3-D transient hydraulic tomography with modular, temporarily-emplaced equipment, Water Resour. Res., 48, W05531, doi:10.1029/2011WR011704, 2012.

Castagna, M. and Bellin, A.: A Bayesian approach for inversion of hydraulic tomographic data, Water Resour. Res., 45, W04410, doi:10.1029/2008WR007078, 2009.

Cooper, H. H. and Jacob, C. E.: A generalized graphical method of evaluating formation constants and summarizing well-field history, US Dept. of the Interior, Geological Survey, Water Resources Division, Ground Water Branch, Washington, D.C., USA, 1946. 
Cressie, N.: Statistics for Spatial Data, in: Wiley Series in Probability and Statistics, Wiley-Interscience, Hoboken, New Jersey, USA, 1993.

de Marsily, G.: Quantitative Hydrogeology: Groundwater Hydrology for Engineers, 1st Edn., Academic Press, Waltham, Massachusetts, USA, 1986.

Deutsch, C. V. and Journel, A. G.: GSLIB, Oxford University Press, Oxford, UK, 1997.

Diggle, P. J. and Ribeiro, P. J.: Model-based Geostatistics, in: Springer Series in Statistics, Springer, New York, USA, 2007.

Evensen, G.: Sequential data assimilation with a nonlinear quasi-geostrophic model using Monte Carlo methods to forecast error statistics, J. Geophys. Res., 99, 10143-10162, doi:10.1029/94JC00572, 1994.

Evensen, G.: Data Assimilation: The Ensemble Kalman Filter, Springer, New York, USA, 2009.

Franssen, H. H. and Kinzelbach, W.: Ensemble Kalman filtering versus sequential self-calibration for inverse modelling of dynamic groundwater flow systems, J. Hydrol., 365, 261-274, doi:10.1016/j.jhydrol.2008.11.033, 2009.

Gottlieb, J. and Dietrich, P.: Identification of the permeability distribution in soil by hydraulic tomography, Inverse Probl., 11, 353360, doi:10.1088/0266-5611/11/2/005, 1995.

Harbaugh, A. W., Banta, E. R., Hill, M. C., and McDonald, M. G.: MODFLOW-2000, The US Geological Survey Modular GroundWater Model-User Guide to Modularization Concepts and the Ground-Water Flow Process, Tech. rep., United States Geological Survey, Reston, Virginia, USA, 2000.

Harvey, C. F. and Gorelick, S. M.: Temporal moment-generating equations: modeling transport and mass transfer in heterogeneous aquifers, Water Resour. Res., 31, 1895-1911, doi:10.1029/95WR01231, 1995.

Illman, W. A., Liu, X., Takeuchi, S., Yeh, T. J., Ando, K., and Saegusa, H.: Hydraulic tomography in fractured granite: Mizunami Underground Research site, Japan, Water Resour. Res., 45, W01406,doi:10.1029/2007WR006715, 2009.

Illman, W. A., Zhu, J., Craig, A. J., and Yin, D.: Comparison of aquifer characterization approaches through steady state groundwater model validation: a controlled laboratory sandbox study, Water Resour. Res., 46, W04502, doi:10.1029/2009WR007745, 2010 .

Illman, W. A., Berg, S. J., and Yeh, T.-C. J.: Comparison of Approaches for Predicting Solute Transport: Sandbox Experiments, Ground Water, 50, 421-431, doi:10.1111/j.17456584.2011.00859.x, 2012

Isaaks, E. H. and Srivastava, R. M.: An Introduction to Applied Geostatistics, Oxford University Press, USA, 1990.

Kalman, R.: A New Approach to Linear Filtering and Prediction Problems, Transactions of the J. Basic Eng.-T. ASME, 3545, available at: http://www.cs.unc.edu/ welch/kalman/media/ pdf/Kalman1960.pdf (last access: 4 March 2014), 1960.

Kitanidis, P. K.: Quasi-linear geostatistical theory for inversing, Water Resour. Res., 31, 2411, doi:10.1029/95WR01945, 1995.

Li, W., Nowak, W., and Cirpka, O. A.: Geostatistical inverse modeling of transient pumping tests using temporal moments of drawdown, Water Resour. Res., 41, W08403, doi:10.1029/2004WR003874, 2005.
Liu, S.: Effectiveness of hydraulic tomography: sandbox experiments, Water Resour. Res., 38, 1034, doi:10.1029/2001WR000338, 2002.

Liu, X. and Kitanidis, P. K.: Large-scale inverse modeling with an application in hydraulic tomography, Water Resour. Res., 47, W02501, doi:10.1029/2010WR009144, 2011.

Liu, X., Illman, W. A., Craig, A. J., Zhu, J., and Yeh, T.-C. J.: Laboratory sandbox validation of transient hydraulic tomography, Water Resour. Res., 43, W05404, doi:10.1029/2006WR005144, 2007.

Mao, D., Yeh, T.-C. J., Wan, L., Wen, J.-C., Lu, W., Lee, C.H., and Hsu, K.-C.: Joint interpretation of sequential pumping tests in unconfined aquifers, Water Resour. Res., 49, 1782-1796, doi:10.1002/wrcr.20129, 2013.

Matheron, G.: Traité de géostatistique appliquée, Mém. Bur. Rech. Géol. Miniéres, Paris, France, 333 pp., 1962.

Moore, C. and Doherty, J.: The cost of uniqueness in groundwater model calibration, Adv. Water Resour., 29, 605-623, doi:10.1016/j.advwatres.2005.07.003, 2006.

Olsthoorn, T. N.: Do a bit more with convolution, Ground Water, 46, 13-22, doi:10.1111/j.1745-6584.2007.00342.x, 2008.

Schöniger, A., Nowak, W., and Hendricks Franssen, H.-J.: Parameter estimation by ensemble Kalman filters with transformed data: approach and application to hydraulic tomography, Water Resour. Res., 48, W04502, doi:10.1029/2011WR010462, 2012.

Shin, V., Lee, Y., and Choi, T.-S.: Generalized Millman's formula and its application for estimation problems, Signal Process., 86, 257-266, doi:10.1016/j.sigpro.2005.05.015, 2006.

Straface, S., Yeh, T.-C. J., Zhu, J., Troisi, S., and Lee, C. H.: Sequential aquifer tests at a well field, Montalto Uffugo Scalo, Italy, Water Resour. Res., 43, W07432, doi:10.1029/2006WR005287, 2007.

Sun, N.-Z.: Inverse Problems in Groundwater Modeling, in: Theory and Applications of Transport in Porous Media, Springer, New York, USA, 1994.

Tarantola, A.: Inverse Problem Theory and Methods for Model Parameter Estimation, SIAM: Society for Industrial and Applied Mathematics, Philadelphia, PA, USA 2004.

Theis, C. V.: The Relation Between the Lowering of the Piezometric Surface and the Rate and Duration of Discharge of a Well Using Ground Water Storage, US Department of the Interior, Geological Survey, Water Resources Division, Ground Water Branch, Washington, D.C., USA, 1935.

Von Asmuth, J. and Maas, K.: The method of impulse response moments: a new method integrating time series-, groundwaterand eco-hydrological modelling, in: Impact of Human Activity on Groundwater Dynamics. Proceedings of a Symposium Held During the Sixth IAHS Scientific Assembly, 18-27 July 2001, Maastricht, Netherlands, 51-58, 2001.

Von Asmuth, J. R., Bierkens, M. F. P., and Maas, K.: Transfer function-noise modeling in continuous time using predefined impulse response functions, Water Resour. Res., 38, 1287, doi:10.1029/2001WR001136, 2002.

Von Asmuth, J. R., Maas, K., Bakker, M., and Petersen, J.: Modeling time series of ground water head fluctuations subjected to multiple stresses, Ground Water, 46, 30-40, doi:10.1111/j.17456584.2007.00382.x, 2008. 
Wu, C.-M., Yeh, T.-C. J., Zhu, J., Lee, T. H., Hsu, N.-S., Chen, C.H., and Sancho, A. F.: Traditional analysis of aquifer tests: comparing apples to oranges?, Water Resour. Res., 41, W09402, doi:10.1029/2004WR003717, 2005.

Yeh, T.-C. J. and Liu, S.: Hydraulic tomography: development of a new aquifer test method, Water Resour. Res., 36, 2095, doi:10.1029/2000WR900114, 2000.

Yeh, T.-C. J. and Zhu, J.: Hydraulic/partitioning tracer tomography for characterization of dense nonaqueous phase liquid source zones, Water Resour. Res., 43, W06435, doi:10.1029/2006WR004877, 2007.
Yin, D. and Illman, W. A.: Hydraulic tomography using temporal moments of drawdown recovery data: a laboratory sandbox study, Water Resour. Res., 45, W01502, doi:10.1029/2007WR006623, 2009.

Zhu, J. and Yeh, T.-C. J.: Characterization of aquifer heterogeneity using transient hydraulic tomography, Water Resour. Res., 41, W07028, doi:10.1029/2004WR003790, 2005.

Zhu, J. and Yeh, T.-C. J.: Analysis of hydraulic tomography using temporal moments of drawdown recovery data, Water Resour. Res., 42, W02403, doi:10.1029/2005WR004309, 2006. 